\title{
ON MULTILINEAR OPERATORS COMMUTING WITH LIE DERIVATIVES
}

\author{
ANDREAS ČAP \\ JAN SLOVÁK
}

\begin{abstract}
Let $E_{1}, \ldots, E_{k}$ and $E$ be natural vector bundles defined over the category $\mathcal{M} f_{m}^{+}$of smooth oriented $m$-dimensional manifolds and orientation preserving local diffeomorphisms, with $m \geq 2$. Let $M$ be an object of $\mathcal{M} f_{m}^{+}$which is connected. We give a complete classification of all separately continuous $k$-linear operators $D: \Gamma_{c}\left(E_{1} M\right) \times \ldots \times \Gamma_{c}\left(E_{k} M\right) \rightarrow \Gamma(E M)$ defined on sections with compact supports, which commute with Lie derivatives, i.e. which satisfy

$$
\mathcal{L}_{X}\left(D\left(s_{1}, \ldots, s_{k}\right)\right)=\sum_{i=1}^{k} D\left(s_{1}, \ldots, \mathcal{L}_{X} s_{i}, \ldots, s_{k}\right),
$$

for all vector fields $X$ on $M$ and sections $s_{j} \in \Gamma_{c}\left(E_{j} M\right)$, in terms of local natural operators and absolutely invariant sections. In special cases we do not need the continuity assumption. We also present several applications in concrete geometrical situations, in particular we give a completely algebraic characterization of some well known Lie brackets.
\end{abstract}

\section{NATURAL OPERATORS}

In this section we give a brief survey over notions and results from the theory of natural bundles and operators which we will need in the sequel and we formulate our main result. Besides the references cited for the results they can all be found in [Kolář-Michor-Slovák].

1.1. Definition. A bundle functor (or natural bundle) on the category $\mathcal{M} f_{m}^{+}$of $m$ dimensional oriented manifolds and orientation preserving smooth locally invertible mappings (so called local diffeomorphisms) is a functor $F: \mathcal{M} f_{m}^{+} \rightarrow \mathcal{F} \mathcal{M}$ with values in fibered manifolds which satisfies:

(i) $B \circ F=\mathrm{id}_{\mathcal{M} f_{m}^{+}}$where $B: \mathcal{F} \mathcal{M} \rightarrow \mathcal{M} f$ is the base functor

(ii) for every inclusion $i: U \rightarrow M$ of an open submanifold, $F U$ is the restriction

$p_{M}^{-1}(U)$ of the value $F M=\left(p_{M}: F M \rightarrow M\right)$ to $U$ and $F i$ is the inclusion

$p_{M}^{-1}(U) \rightarrow F M$.

A vector bundle functor (or natural vector bundle) is a bundle functor with values in the category of finite dimensional vector bundles and fiberwise invertible vector bundle homomorphisms.

1991 Mathematics Subject Classification. 53A55, 58A20.

Key words and phrases. natural operators, natural bundles, Lie differentiation.

First author supported by project P 77724 PHY of 'Fonds zur Förderung der wissenschaftlichen Forschung'. Second author supported by a grant of the GAČR. 
1.2. By the general theory, cf. [Palais-Terng], [Epstein-Thurston], each natural bundle is obtained (up to isomorphisms) by the construction of associated bundles to the (higher order) frame bundles $P^{r^{+}} M$. The latter principal bundles are defined as the bundles of $r$-jets of locally defined orientation preserving diffeomorphisms $\mathbb{R}^{m} \rightarrow M$ at the origin $0 \in \mathbb{R}^{m}$, and their structure groups are the Lie groups of $r$-jets of orientation preserving diffeomorphisms $\mathbb{R}^{m} \rightarrow \mathbb{R}^{m}$ which keep the origin fixed, the so called jet groups. Then the values of $F$ on morphisms depend only on $r$-jets for some non-negative integer $r$, which is called the order of $F$.

In particular, all natural bundles transform smoothly parameterized families of morphisms into smoothly parameterized families (the so called regularity). Hence we can apply a bundle functor $F$ to flows of vector fields on $M$ and we get flows on the values $F M$. This gives rise to the so called flow operator which transforms vector fields on $M$ into vector fields on $F M$.

Vector bundle functors correspond to linear representations of the jet groups in finite dimensional vector spaces and the flow operators can be used to define Lie derivatives of sections of a natural vector bundle analogously to the classical case of tensor bundles, cf. [Terng] and [Čap-Slovák]. By the regularity of vector bundle functors, the Lie derivatives define a continuous action of the Lie algebra of vector fields on the space of sections. This action is local in both arguments and so in view of the localization property of the vector bundle functors we get for each vector bundle functor $F$ an action of the sheaf of vector fields on an oriented manifold $M$ on the sheaf of local sections of $F M$.

1.3. Absolutely invariant sections. Let $F$ be a natural vector bundle defined on the category $\mathcal{M} f_{m}^{+}$and let $M$ be an object of $\mathcal{M} f_{m}^{+}$which is connected. A section $s \in \Gamma(F M)$ is called absolutely invariant if and only if $\mathcal{L}_{X} s=0$ for all vector fields $X$ on $M$. The finiteness of the order of the bundle functor $F$ and arguments from the proof of the main theorem of [Čap-Slovák] imply that $s$ is then invariant for the actions of all orientation preserving diffeomorphisms of $M$. Now assume that $F$ is of order $r$ and let $\rho: G_{m}^{r+} \rightarrow G L(V)$ be the corresponding representation of the jet group. Then one easily verifies that the absolutely invariant sections of $F M$ are in bijective correspondence with elements of $V$ which are invariant for the action $\rho$. In particular we see that the absolutely invariant sections always form a finite dimensional vector space of dimension at most the fiber dimension of $F M$, and that an absolutely invariant section is determined by its restriction to one point. Moreover an absolutely invariant section $s$ of $F M$ gives rise to a unique absolutely invariant section of $F N$ for any oriented $m$-dimensional manifold $N$.

Note that in many concrete situations there exist no absolutely invariant sections. For example in a subbundle of a tensor bundle $\otimes^{p} T M \otimes \otimes^{q} T^{*} M$ absolutely invariant sections can exist only if $p=q$.

1.4. Definition. Let $E, F: \mathcal{M} f_{m}^{+} \rightarrow \mathcal{F M}$ be two bundle functors. A natural operator $D: E \rightarrow F$ is a system $D_{M}$ of local smooth operators, for all oriented $m$-dimensional manifolds $M$, which commute with the actions of local diffeomorphisms. So $D_{M}: \Gamma(E M) \rightarrow \Gamma(F M)$ transforms smooth families of sections of $F M$ into smooth families of sections of $G M$ and $D_{M}(s)(x)$ depends only on the germ of $s$ at $x$ for all sections $s \in \Gamma(E M)$ and points $x \in M$. Moreover for any diffeomorphism $\varphi: M \rightarrow N$ we have $F(\varphi)_{*} \circ D_{M}=D_{N} \circ E(\varphi)_{*}: \Gamma(E M) \rightarrow \Gamma(F N)$, and for any open submanifold $U \subset M$ and section $s \in \Gamma(E M)$ we have $D_{U}\left(\left.s\right|_{U}\right)=\left.D_{M}(s)\right|_{U}$. 
Each local $k$-linear natural operator $D: E_{1} \times \ldots \times E_{k} \rightarrow E$ satisfies

$$
\mathcal{L}_{X}\left(D_{M}\left(s_{1}, \ldots, s_{k}\right)\right)=\sum_{i=1}^{k} D_{M}\left(s_{1}, \ldots, \mathcal{L}_{X} s_{i}, \ldots, s_{k}\right)
$$

for each manifold $M$, all vector fields $X$ on $M$ and all $s_{i} \in \Gamma\left(E_{i} M\right)$. We say that $D$ commutes with Lie derivatives. Moreover, each $k$-linear local operator $D_{M}: \Gamma\left(E_{1} M\right) \times \ldots \times \Gamma\left(E_{k} M\right) \rightarrow \Gamma(E M)$ which commutes with Lie derivatives extends to a unique natural operator $D$, see [Čap-Slovák, 3.2].

1.5. Determination of local natural operators. Let us consider a $k$-linear natural operator $D: E_{1} \times \ldots \times E_{k} \rightarrow E$, where the $E_{i}$ and $E$ are natural vector bundles. By the multilinear Peetre theorem (c.f. [Cahen-de Wilde-Gutt] or [Slovák]) $D$ is of some finite order $r$. Let $J^{r} E_{i}$ denote the $r$-jet prolongations of the natural vector bundles $E_{i}$, let $V_{i}$ and $V$ be the standard fibers of the $E_{i}$ and $E$, and let $T_{m}^{r} V_{i}$ be the standard fiber of $J^{r} E_{i}$. The natural operator $D$ is uniquely determined by its value on $\mathbb{R}^{m}$ and $D_{\mathbb{R}^{m}}$ is induced by a $k$-linear vector bundle homomorphism $J^{r} E_{1} \mathbb{R}^{m} \times \ldots \times J^{r} E_{k} \mathbb{R}^{m} \rightarrow E \mathbb{R}^{m}$. This in turn is uniquely determined by the induced linear map $\tilde{D}: T_{m}^{r} V_{1} \otimes \cdots \otimes T_{m}^{r} V_{k} \rightarrow V$. The spaces $T_{m}^{r} V_{i}$ and $V$ are modules over the jet group $G_{m}^{r+\ell}$, where $\ell$ is the maximum of the orders of the bundle functors $E_{i}$ and $E$, and the fact that $\tilde{D}$ comes from a natural operator is equivalent to equivariancy of $\tilde{D}$ for these actions.

Thus the determination of natural operators of fixed order $r$ reduces to the classification of equivariant linear maps between two finite dimensional representations of the jet group $G_{m}^{r+\ell}$. This classification has been carried out in a variety of special cases, see [Kolár-Michor-Slovák] for a collection of results in this direction.

In particular the space of multilinear local natural operators of fixed order between fixed bundles is always finite dimensional. Moreover we can prove that there is always some maximal order for such operators:

1.6. Lemma. Let $E_{1}, \ldots, E_{k}$ and $E$ be vector bundle functors on $\mathcal{M} f_{m}^{+}$. Then the space of all $k$-linear (local) natural operators $D: E_{1} \times \ldots \times E_{k} \rightarrow E$ is finite dimensional.

Proof. As discussed above, each such operator $D_{M}: \Gamma\left(E_{1} M\right) \oplus \cdots \oplus \Gamma\left(E_{k} M\right) \rightarrow$ $\Gamma(E M)$ factors through a $k$-linear $G_{m}^{r+\ell}$-equivariant map on the jets of sections in one point of $\mathbb{R}^{m}, \tilde{D}: T_{m}^{r} V_{1} \otimes \cdots \otimes T_{m}^{r} V_{k} \rightarrow V$, where $r$ is the order of the operator, $\ell$ is the maximum of the orders of the natural bundles. Let us write $D^{*}: V^{*} \rightarrow$ $\left(T_{m}^{r} V_{1}\right)^{*} \otimes \cdots \otimes\left(T_{m}^{r} V_{k}\right)^{*}$ for the dual linear mapping to the corresponding linear mapping on the tensor product of the jet spaces. The jet group $G_{m}^{1}=G L(m, \mathbb{R})$ of order one is always a subgroup and thus, in particular the mapping $D^{*}$ is an $S L(m, \mathbb{R})$-module homomorphism. As representation spaces for $S L(m, \mathbb{R})$, all the standard fibers $V_{1}, \ldots, V_{k}$ and $V$ decompose into irreducible representations, as well as the whole tensor product of the duals. Each of the irreducible components is determined by a highest weight vector sitting in the whole space, we shall write $V_{\lambda}$ for a component with highest weight $\lambda$. Furthermore, we always have $T_{m}^{r} W=$ $\oplus_{i=0}^{r} W \otimes S^{i} \mathbb{R}^{m *}$ as $G L(m, \mathbb{R})$-modules, in particular $T_{m}^{r}\left(W_{1} \oplus W_{2}\right)=T_{m}^{r} W_{1} \oplus T_{m}^{r} W_{2}$ for any two $G L(m, \mathbb{R})$-modules. Thus we may rewrite the target space of $D^{*}$ in 
the form

$$
\begin{aligned}
\bigotimes_{i=1}^{k}\left(\bigoplus_{j=0}^{r} V_{i}^{*} \otimes S^{j} \mathbb{R}^{m}\right) & =\bigoplus_{s=1}^{N} \bigoplus_{j_{1}, \ldots, j_{k}=0}^{r}\left(V_{\rho_{s}} \otimes \otimes_{i=1}^{k} S^{j_{i}} \mathbb{R}^{m}\right) \\
& \subset \bigoplus_{s=1}^{N} \bigoplus_{j_{1}, \ldots, j_{k}=0}^{r}\left(V_{\rho_{s}} \otimes \otimes^{j_{1}+\cdots+j_{k}} \mathbb{R}^{m}\right)
\end{aligned}
$$

where the spaces $V_{\rho_{s}}$ are the irreducible components in the tensor product $V_{1}^{*} \otimes$ $\cdots \otimes V_{k}^{*}$. Since the decomposition into irreducible components is always unique up to order and since a homomorphism between irreducible components is a multiple of the identity, the mapping $D^{*}$ is just the inclusion of the irreducible components of $V^{*}=\oplus_{i} V_{\mu_{i}}$ into the tensor product. Thus we have only to show that highest weight vectors in the tensor product with the weights $\mu_{i}$ can appear only in the components $V_{\rho_{s}} \otimes \otimes^{j} \mathbb{R}^{m}$ with $j$ less that some fixed bound.

If our jet spaces were completely reducible $G L(m, \mathbb{R})$-modules, we could apply just the above discussion and to compare the actions of the center of $G L(m, \mathbb{R})$ on the domain and the target. The multiples of the identity matrix in the Lie algebra $\mathfrak{g l}(m, \mathbb{R})$ must act by some scalar and each copy of $\mathbb{R}^{m}$ in the tensor product increases this scalar by one. This simple idea leads then directly to a bound as required. Since we do not want to restrict ourselves to completely decomposable $G L(m, \mathbb{R})$-modules, we have to use the above decomposition into irreducible $S L(m, \mathbb{R})$-modules and to apply the equivariancy with respect to the induced action of the universal enveloping algebra of $\mathfrak{s l}(m, \mathbb{R})$, where the center is not trivial.

Let us assume there is a highest weight vector with weight $\mu$ in $V_{\rho} \otimes \otimes^{j} \mathbb{R}^{m}$. Then we can complexify both the representation spaces, and we shall find a highest weight vector with weight $\mu$ in the $\mathfrak{U}\left(\mathfrak{s l}(m, \mathbb{C})\right.$ )-module $V_{\rho_{s}} \otimes \otimes^{j} \mathbb{C}^{m}$ (now $V_{\rho_{s}}$ means the complex irreducible representation with highest weight $\rho_{s}$ ).

For the semisimple Lie algebra $\mathfrak{s l}(m, \mathbb{C})$, there is the Casimir operator $C$ sitting in the center of $\mathfrak{U}(\mathfrak{s l}(m, \mathbb{C}))$ and so acting by a scalar $C_{\mu}$ on each irreducible representation space $V_{\mu}$. This scalar can be computed easily by means of the highest weights, $C_{\mu}=\langle\mu, \mu+2 \delta\rangle$ where $2 \delta$ is the sum of all positive roots and $\langle$,$\rangle is the$ Killing form on the algebra, see e.g. [Samelson, p. 121]. In our case, the weights are in the dual to the Cartan algebra which consists of trace free diagonal matrices. If $e^{1}, \ldots, e^{m}$ is the usual linear basis, the positive roots are $e^{i}-e^{j}$ with $i<j$, all possible highest weights are of the form $a_{1} e^{1}+\cdots+a_{m-1} e^{m-1}$ with integers $a_{1} \geq a_{2} \geq$ $\cdots \geq a_{m-1} \geq 0$, and the Killing form is just the Euclidean metric up to a constant negative multiple. In particular we get $\delta=(m-1) e^{1}+(m-2) e^{2}+\cdots+e^{m-1}$.

In order to estimate $C_{\mu}$ for our irreducible component in the tensor product, we have to know which weights may appear in a tensor product of two irreducible representations. A general answer is obtained easily by means of some of the well known consequences of the Weyl's character formula, e.g. Klimyk's formula, see [Samelson, p. 128]: We have only to consider the highest weight of the first representation and to add any weight of the other one (only those which yield dominant weights can apply and the multiplicity must be computed). In our case this means, we take the weight $\rho$ and we add, step by step, the weights of $\mathbb{C}^{m}$. To get all of them, we apply first the Weyl group to the highest weight $e^{1}$ of $\mathbb{C}^{m}$ which yields just the weights $e^{1}, \ldots, e^{m}$. These must be involved, but the dimension of the 
sum of the corresponding weight spaces is at least $m$, so we are done. Altogether, a highest weight in $V_{\rho} \otimes \otimes^{j} \mathbb{R}^{m}$ must be of the form $\rho+e^{i_{1}}+\cdots+e^{i_{j}}$ and we obtain

$$
\begin{aligned}
C_{\mu} & =\left\langle\rho+e^{i_{1}}+\cdots+e^{i_{j}}, \rho+e^{i_{1}}+\cdots+e^{i_{j}}+2\left((m-1) e^{1}+\cdots+e^{m-1}\right)\right\rangle \\
& =C_{\rho}+2\left\langle\rho, e^{i_{1}}+\cdots+e^{i_{j}}\right\rangle+\left\langle e^{i_{1}}+\cdots+e^{i_{j}}, e^{i_{1}}+\cdots+e^{i_{j}}+2 \delta\right\rangle \\
& \leq \text { const - a positive constant multiple of } j .
\end{aligned}
$$

This estimate implies that for each fixed $\rho$ and $\mu$, the order $j$ is bounded and the lemma is proved.

In fact the proof of lemma 1.6 shows even that the same statement holds for vector bundle functors and operators defined on the category of smooth oriented $m$-dimensional manifolds with fixed volume forms and volume preserving local diffeomorphisms.

Next we introduce a class of non-local operators which commute with Lie derivatives.

1.7. Definition. Let $E_{1}, \ldots, E_{k}, E$ be natural vector bundles defined on the category $\mathcal{M} f_{m}^{+}$, and let us write $\Gamma_{c}\left(E_{i} M\right)$ for the space of compactly supported smooth sections of the vector bundle $E_{i} M$ over an $m$-dimensional oriented manifold $M$ while $\Gamma(E M)$ means the space of all smooth sections. Let $I^{1}=\left\{i_{1}^{1}<\cdots<i_{n_{1}}^{1}\right\}, \ldots, I^{r}=$ $\left\{i_{1}^{r}<\cdots<i_{n_{r}}^{r}\right\}, J=\left\{j_{1}<\cdots<j_{n}\right\}$ with $i_{1}^{1}<\cdots<i_{1}^{r}$ be a partition of the set $\{1, \ldots, k\}$ into disjoint subsets, such that all $I^{j}$ are nonempty, while $J$ is allowed to be empty.

An elementary almost natural operator $\Gamma_{c}\left(E_{1} M\right) \times \ldots \times \Gamma_{c}\left(E_{k} M\right) \rightarrow \Gamma(E M)$ of type $\left(I^{1}, \ldots, I^{r}, J\right)$ is an operator of the form

$$
\left(s_{1}, \ldots s_{k}\right) \mapsto \lambda^{1}\left(s_{i_{1}^{1}}, \ldots, s_{i_{n_{1}}^{1}}\right) \ldots \lambda^{r}\left(s_{i_{1}^{r}}, \ldots, s_{i_{n_{r}}^{r}}\right) D_{M}\left(s_{j_{1}}, \ldots, s_{j_{n}}\right),
$$

where $D: E_{j_{1}} \times \ldots \times E_{j_{n}} \rightarrow E$ is a $j_{n}$-linear local natural operator and any $\lambda^{\ell}$ is an operator $\Gamma_{c}\left(E_{i_{1}^{\ell}}\right) \times \ldots \times \Gamma_{c}\left(E_{i_{n_{\ell}}^{\ell}}\right) \rightarrow \mathbb{R}$ of the form

$$
\left(s_{1}, \ldots, s_{n_{\ell}}\right) \mapsto \int_{M}\left\langle D_{M}^{\ell}\left(s_{1}, \ldots, s_{n_{\ell}-1}\right), s_{n_{\ell}}\right\rangle,
$$

where $D^{\ell}: E_{i_{1}^{\ell}} \times \ldots \times E_{i_{n_{\ell}-1}^{\ell}} \rightarrow E_{i_{n_{\ell}}^{\ell}}^{*} \otimes \Lambda^{m} T^{*}$ is a $\left(n_{\ell}-1\right)$-linear local natural operator and $\langle\quad, \quad\rangle$ denotes the canonical pairing $\Gamma_{c}\left(E_{i_{n_{\ell}}^{\ell}}^{*} M \otimes \Lambda^{m} T^{*} M\right) \times \Gamma_{c}\left(E_{i_{n_{\ell}}^{\ell}} M\right) \rightarrow$ $\Gamma_{c}\left(\Lambda^{m} T^{*} M\right)$. In this notation we use the convention that a local natural operator without arguments is just an absolutely invariant section of the target bundle.

An almost natural operator $E_{1} M \times \ldots \times E_{k} M \rightarrow E M$ is a linear combination of elementary almost natural operators.

1.8. Proposition. Any almost natural operator commutes with Lie derivatives.

Proof. Let us first show that for a multilinear map $\lambda$ as in definition 1.7 we have $\sum_{i=1}^{n} \lambda\left(s_{1}, \ldots, \mathcal{L}_{X} s_{i}, \ldots, s_{n}\right)=0$ for any sections $s_{i}$ and any vector field $X$ on $M$. Since any Lie derivative of an $m$-form on an $m$-dimensional manifold is an exact 
form we have:

$$
\begin{aligned}
0= & \int_{M} \mathcal{L}_{X}\left(\left\langle D_{M}\left(s_{1}, \ldots, s_{n-1}\right), s_{n}\right\rangle\right)= \\
= & \int_{M}\left(\left\langle\mathcal{L}_{X}\left(D_{M}\left(s_{1}, \ldots, s_{n-1}\right)\right), s_{n}\right\rangle+\left\langle D_{M}\left(s_{1}, \ldots, s_{n-1}\right), \mathcal{L}_{X} s_{n}\right\rangle\right)= \\
= & \sum_{i=1}^{n-1} \int_{M}\left\langle D_{M}\left(s_{1}, \ldots, \mathcal{L}_{X} s_{i}, \ldots, s_{n-1}\right), s_{n}\right\rangle+ \\
& \quad+\int_{M}\left\langle D_{M}\left(s_{1}, \ldots, s_{n-1}\right), \mathcal{L}_{X} s_{n}\right\rangle= \\
= & \sum_{i=1}^{n} \lambda\left(s_{1}, \ldots, \mathcal{L}_{X} s_{i}, \ldots, s_{n}\right)
\end{aligned}
$$

From this the result is obvious.

The aim of this paper is to discuss to what extent the converse of this proposition holds, i.e. whether all multilinear operators which commute with Lie derivatives are almost natural.

The space of sections of a vector bundle has a canonical Fréchet topology (in principle just uniform convergence in each derivative), while the space of compactly supported sections of a bundle can be canonically topologized as the inductive limit of the spaces of sections having support in some fixed compact subset, which are again Fréchet spaces (see 2.2 for a more detailed description). Obviously any almost natural operator is jointly continuous for these topologies, i.e. continuous as a mapping $D: \Gamma_{c}\left(E_{1} M\right) \times \ldots \times \Gamma_{c}\left(E_{k} M\right) \rightarrow \Gamma(E M)$.

The main result of this paper is that under the weaker assumption of continuity in each argument separately, the converse to 1.8 is actually true:

1.9. Theorem. Let $E_{1}, \ldots, E_{k}, E$ be natural vector bundles defined on the category $\mathcal{M} f_{m}^{+}, m \geq 2$, and let $M$ be a connected oriented smooth manifold of dimension $m$. Then any separately continuous $k$-linear operator $D: \Gamma_{c}\left(E_{1} M\right) \times \ldots \times \Gamma_{c}\left(E_{k} M\right) \rightarrow$ $\Gamma(E M)$ which commutes with Lie derivatives is an almost natural operator. In particular any such operator is automatically jointly continuous and the space of such operators is always finite dimensional and independent of the manifold $M$.

Let us remark that the assumption of separate continuity is needed in one step in the proof, the application of the Schwartz kernel theorem in 3.3. In our opinion, the only way to avoid the continuity assumption is to prove that continuity already follows from the commutation with Lie derivatives. Certainly this is a rather difficult problem, since there even are examples of (non-linear) local natural operators which are not continuous, see e.g. [Kolář-Michor-Slovák, 23.9].

\section{NATURAL PREShEAVES}

Although our results apply mainly to natural vector bundles and their sections we have to use the more technical notion of an admissible natural presheaf in the proofs.

2.1. Definitions. Let $M$ be a smooth manifold. By $\mathcal{X}(\quad)$ we denote the sheaf of smooth vector fields over $M$. 
(1) A natural presheaf $\mathcal{F}$ on $M$ is a presheaf of locally convex modules over the sheaf of Lie algebras $\mathcal{X}(M)$. This means that for any open subset $U$ of $M$ there is a locally convex vector space $\mathcal{F}(U)$ on which the Lie algebra $\mathcal{X}(U)$ acts by continuous linear operators, which we denote by $\mathcal{L}_{X}$ for $X \in \mathcal{X}(U)$. By the same symbol we denote the actions of the restrictions of $X$ to open subsets of $U$ on the corresponding spaces. Moreover for any open subset $V \subset U$ there is a continuous linear restriction mapping $r_{V}^{U}: \mathcal{F}(U) \rightarrow \mathcal{F}(V)$ which is equivariant over the corresponding restriction map for vector fields for the Lie algebra actions.

This is an infinitesimal version of the definition of a natural presheaf given in [Eck].

(2) An element $s \in \mathcal{F}(U)$ is called absolutely invariant if and only if $\mathcal{L}_{X} s=0$ for all $X \in \mathcal{X}(M)$ with support contained in $U$.

(3) A natural presheaf $\mathcal{F}$ over $M$ is called admissible if and only if the following two conditions are satisfied:

(i) For any open subset $U$ of $M$ and system of countably many open sets $\left\{U_{i}\right\}$ such that $U_{i} \subset U_{i+1}$ for all $i$, and such that $U=\cup_{i \in \mathbb{N}} U_{i}$, each element of $\mathcal{F}(U)$ is uniquely determined by its restrictions to all spaces $\mathcal{F}\left(U_{i}\right)$.

(ii) For any connected open subset $U$ of $M$ and any absolutely invariant element $s \in \mathcal{F}(U)$ there is a unique absolutely invariant element $\tilde{s} \in \mathcal{F}(M)$ such that $r_{U}^{M}(\tilde{s})=s$. Moreover the operator of extending absolutely invariant elements is continuous.

(4) Let $E_{1} \rightarrow M, \ldots, E_{k} \rightarrow M$ be vector bundles, $\mathcal{F}$ a presheaf of vector spaces over $M$ and let us write $\Gamma_{c}\left(E_{i}\right)$ for the space of sections of $E_{i}$ with compact support. A $k$-linear operator $D: \Gamma_{c}\left(E_{1}\right) \times \ldots \times \Gamma_{c}\left(E_{k}\right) \rightarrow \mathcal{F}(M)$ is called local if and only if for each open subset $U$ of $M$ and each $i, 1 \leq i \leq k$, the condition $\left.s_{i}\right|_{U}=0$ implies that $r_{U}^{M}\left(D\left(s_{1}, \ldots, s_{k}\right)\right)=0$.

(5) Let $E_{1} \rightarrow M, \ldots, E_{k} \rightarrow M$ be vector bundles, $V$ a vector space. A $k$-linear map $\lambda: \Gamma_{c}\left(E_{1}\right) \times \ldots \times \Gamma_{c}\left(E_{k}\right) \rightarrow V$ is called weakly local if and only if $\lambda\left(s_{1}, \ldots, s_{k}\right)=0$ for all sections with $\operatorname{supp}\left(s_{1}\right) \cap \cdots \cap \operatorname{supp}\left(s_{k}\right)=\emptyset$.

\subsection{Topologies on spaces of sections and operators between them.}

Before we give examples of natural presheaves we describe the topologies we will use. For an open subset $U \subset \mathbb{R}^{m}$ we put on $C^{\infty}\left(U, \mathbb{R}^{n}\right)$ as usual the topology of uniform convergence in all derivatives separately, which is a Fréchet topology. Next for a smooth vector bundle $E \rightarrow M$ over a smooth manifold a vector bundle atlas $\left(U_{i}\right)$ gives rise to linear maps $\Gamma(E) \rightarrow C^{\infty}\left(U_{i}, \mathbb{R}^{n}\right)$, where $n$ is the fiber dimension of $E$, and we put on $\Gamma(E)$ the initial topology with respect to these maps. Since we can use a countable atlas $\Gamma(E)$ is again a Fréchet space. Next for any compact subset $K \subset M$ the space $\Gamma_{c}(E ; K)$ of all sections of $E$ with support contained in $K$ is a closed linear subspace of $\Gamma(E)$ and thus also a Fréchet space with the induced topology. Now for $i \in \mathbb{N}$ we can choose compact subsets $K_{i} \subset M$ such that $K_{i} \subset K_{i+1}$ and such that $M=\cup_{i \in \mathbb{N}} K_{i}$. Then $\Gamma_{c}\left(E ; K_{i}\right)$ is a closed linear subspace of $\Gamma_{c}\left(E ; K_{i+1}\right)$ and $\Gamma_{c}(E)=\cup_{i \in \mathbb{N}} \Gamma_{c}\left(E ; K_{i}\right)$ as a set. We topologize $\Gamma_{c}(E)$ as the inductive limit of the spaces $\Gamma_{c}\left(E ; K_{i}\right)$, so $\Gamma_{c}(E)$ is the strict inductive limit of a sequence of Fréchet spaces. In particular this implies that $\Gamma_{c}(E)$ is a bornological space, i.e. a linear map from $\Gamma_{c}(E)$ to any locally convex vector space is continuous if and only if it is bounded.

Consider the space $L\left(\Gamma_{c}\left(E_{1}\right), \ldots, \Gamma_{c}\left(E_{k}\right) ; \Gamma(E)\right)$ of all bounded $k$-linear maps $\Gamma_{c}\left(E_{1}\right) \times \ldots \times \Gamma_{c}\left(E_{k}\right) \rightarrow \Gamma(E)$, where the $E_{i}$ and $E$ are vector bundles over $M$. 
Since $\Gamma_{c}\left(E_{i}\right)$ is bornological we conclude from [Frölicher-Kriegl, 3.7.5] that the bounded $k$-linear maps are exactly the separately continuous ones.

Now we declare a subset $B \subset L\left(\Gamma_{c}\left(E_{1}\right), \ldots, \Gamma_{c}\left(E_{k}\right) ; \Gamma(E)\right)$ to be bounded if and only if $B\left(A_{1} \times \ldots \times A_{k}\right)$ is bounded in $\Gamma(E)$ for all bounded subsets $A_{i} \subset \Gamma_{c}\left(E_{i}\right)$. Then on $L\left(\Gamma_{c}\left(E_{1}\right), \ldots, \Gamma_{c}\left(E_{k}\right) ; \Gamma(E)\right)$ we put the associated locally convex topology, i.e. we take as a basis of neighborhoods of zero all absolutely convex subsets $U$ such that for any bounded subset $B$ there exists some $t \in \mathbb{R}$ such that $B \subset t \cdot U$. Note that by definition this is a bornological topology. Moreover by [Frölicher-Kriegl, 3.7.3] flipping coordinates gives an isomorphism

$$
\begin{aligned}
L\left(\Gamma_{c}\left(E_{1}\right), \ldots, \Gamma_{c}\left(E_{k}\right)\right. & ; \Gamma(E)) \cong \\
& \cong L\left(\Gamma_{c}\left(E_{1}\right), \ldots, \Gamma_{c}\left(E_{\ell}\right) ; L\left(\Gamma_{c}\left(E_{\ell+1}\right), \ldots, \Gamma_{c}\left(E_{k}\right) ; \Gamma(E)\right)\right) .
\end{aligned}
$$

2.3. Examples of natural presheaves. (1) Let $E$ be a vector bundle functor defined on the category $\mathcal{M} f_{m}$ of $m$-dimensional smooth manifolds and local diffeomorphisms. Then with the usual notion of the Lie derivative along vector fields and the topology described above, the spaces $\Gamma(E U)$ of smooth sections of $E U$ form a natural presheaf (in fact a natural sheaf) over $M$. In section 4 we will prove that this natural presheaf is admissible.

(2) A less simple example which will be crucial in the sequel is the following: Let $E_{1}, \ldots, E_{k}, E$ be vector bundle functors as above. Put

$$
\mathcal{F}(U):=L\left(\Gamma_{c}\left(E_{1} U\right), \ldots, \Gamma_{c}\left(E_{k} U\right) ; \Gamma(E U)\right)
$$

the space of all separately continuous $k$-linear operators. On this space we define the action of the Lie algebra $\mathcal{X}(U)$ by

$$
\left(\mathcal{L}_{X} D\right)\left(s_{1}, \ldots, s_{k}\right):=\mathcal{L}_{X}\left(D\left(s_{1}, \ldots, s_{k}\right)\right)-\sum_{i=1}^{k} D\left(s_{1}, \ldots, \mathcal{L}_{X} s_{i}, \ldots, s_{k}\right)
$$

This action is continuous since it is just given by compositions with continuous linear operators. To define the restriction mappings just note that for open sets $V \subset U$ any section with compact support of the restricted vector bundle over $V$ can be extended by zero to a section over $U$. It will be shown in section 5 that this natural presheaf is also admissible. Note that the absolutely invariant elements in $\mathcal{F}(U)$ are by definition exactly those operators which commute with Lie derivatives.

2.4. Remark. When dealing with operators with values in spaces of operators one has to be very careful about the various meanings of locality and weak locality. Moreover the name 'weakly local' is a little misleading when dealing with operators with values in general admissible natural presheaves. Clearly any local operator with values in a sheaf over $M$ is a weakly local mapping. But if one deals with presheaves which only satisfy the very weak condition (i) of 2.1.(3) then there are local operators which are not weakly local. Let us discuss this in an example which is relevant in the sequel:

For a smooth manifold $M$ of dimension $m$ consider the trilinear operator

$$
D: C_{c}^{\infty}(M, \mathbb{R}) \times C_{c}^{\infty}(M, \mathbb{R}) \times \Omega_{c}^{m}(M) \rightarrow C^{\infty}(M, \mathbb{R})
$$


defined by $D(f, g, \omega):=f \cdot \int_{M} g \cdot \omega$. As a trilinear operator this is neither local nor weakly local. But we can consider $D$ in three ways as a linear operator with values in a space of bilinear operators. In fact all these associated linear operators are local, since this just means that if one of the three sections $f, g$ and $\omega$ vanishes on an open subset $U$ and the other two sections have support contained in $U$ then $D(f, g, \omega)$ vanishes on $U$ and this is clearly satisfied.

Next we can consider $D$ in three ways as a bilinear operator with values in a space of linear operators. In particular consider the operator $(f, g) \mapsto(\omega \mapsto$ $D(f, g, \omega))$. Then this operator is local since if either $f$ or $g$ vanish on an open subset $U$ of $M$ and $\omega$ has support contained in $U$ then $D(f, g, \omega)$ vanishes on $U$. On the other hand this operator is not weakly local since $f$ and $g$ having disjoint supports does not imply that $D(f, g, \omega)=0$. Similarly one checks that the operator $(f, \omega) \mapsto(g \mapsto D(f, g, \omega))$ is local but not weakly local while on the other hand $(g, \omega) \mapsto(f \mapsto D(f, g, \omega))$ is weakly local but not local.

2.5. Let $E_{1}, \ldots, E_{k}$ be vector bundle functors defined on the category $\mathcal{M} f_{m}^{+}, m \geq$ 2 , and let $\mathcal{F}$ be an admissible natural presheaf over an oriented connected $m^{-}$ dimensional manifold $M$. Let $D: \Gamma_{c}\left(E_{1} M\right) \times \ldots \times \Gamma_{c}\left(E_{k} M\right) \rightarrow \mathcal{F}(M)$ be a $k$-linear operator which commutes with Lie derivatives, i.e. such that

$$
\mathcal{L}_{X}\left(D\left(s_{1}, \ldots, s_{k}\right)\right)=\sum_{i=1}^{k} D\left(s_{1}, \ldots, \mathcal{L}_{X} s_{i}, \ldots, s_{k}\right)
$$

for all $X \in \mathcal{X}(M), s_{i} \in \Gamma_{c}\left(E_{i} M\right)$.

First consider sections $s_{i} \in \Gamma_{c}\left(E_{i} M\right)$ and suppose that over some open set $U$ all $s_{i}$ are identically zero. Then $\mathcal{L}_{X} s_{i}$ is globally zero for all vector fields $X$ with support in $U$ and so the commutation with Lie derivatives implies that the restriction of $D\left(s_{1}, \ldots, s_{k}\right)$ to $U$ is absolutely invariant.

Lemma. Suppose that $W:=M \backslash\left(\cup_{i} \operatorname{supp}\left(s_{i}\right)\right) \neq \emptyset$. Then there is a unique absolutely invariant element $D\left(s_{1}, \ldots, s_{k}\right)_{\mathrm{inv}} \in \mathcal{F}(M)$ such that $r_{W}^{M}\left(D\left(s_{1}, \ldots, s_{k}\right)_{\mathrm{inv}}\right)=$ $r_{W}^{M}\left(D\left(s_{1}, \ldots, s_{k}\right)\right)$.

Proof. Since $\mathcal{F}$ is admissible, the absolutely invariant restrictions of $D\left(s_{1}, \ldots, s_{k}\right)$ to the individual connected components of $W$ extend uniquely to globally defined absolutely invariant elements. Thus, we only have to show that for any two connected components $U$ and $V$ of $W$ the two absolutely invariant elements in $\mathcal{F}(M)$ determined by the restrictions of $D\left(s_{1}, \ldots, s_{k}\right)$ to $U$ and $V$ coincide. Since $M$ is connected and of dimension at least 2 we can choose $k+1$ smooth curves $c_{1}, \ldots, c_{k+1}:[0,1] \rightarrow M$, which all start in $U$, end in $V$ and are pairwise disjoint. Then there are open sets $U_{i}$ and $V_{i}$ for $1 \leq i \leq k+1$ such that $c_{i}([0,1]) \subset U_{i} \subset \bar{U}_{i} \subset V_{i}$ and such that the sets $V_{i}$ are pairwise disjoint. Next for each $i=1, \ldots, k$ choose a smooth function $f_{i} \in C^{\infty}(M,[0,1])$ which is identically 1 on $\bar{U}_{i}$ and vanishes outside of $V_{i}$ and put $f_{k+1}:=1-\sum_{i=1}^{k} f_{i}$.

By $k$-linearity $D\left(s_{1}, \ldots, s_{k}\right)=\sum_{I} D\left(f_{i_{1}} s_{1}, \ldots, f_{i_{k}} s_{k}\right)$, where $I$ runs over the set of all multiindices $\left(i_{1}, \ldots, i_{k}\right)$ with $1 \leq i_{j} \leq k+1$. But now observe that for any such multiindex there is at least one integer $\ell, 1 \leq \ell \leq k+1$, which does not appear in the multiindex. Thus all sections $f_{i_{1}} s_{1}, \ldots, f_{i_{k}} s_{k}$ vanish locally around the curve $c_{\ell}$ and hence on a connected open set which intersects both $U$ and $V$. So for each of the summands the two extensions coincide and hence the same is true for the sum. 
2.6. For $i=1, \ldots, k$ choose nonempty open subsets $U_{i}$ and $V_{i}$ of $M$ such that $U_{i} \subset \bar{U}_{i} \subset V_{i}$ and such that the sets $\bar{V}_{i}$ are pairwise disjoint. Since $M$ is connected the open subset $U_{k+1}:=M \backslash\left(\cup_{i} \bar{V}_{i}\right)$ is nonempty. Clearly it is possible to find such a configuration with all $V_{i}$ contained in an arbitrary small open subset of $M$. Then choose smooth functions $f_{i} \in C^{\infty}(M,[0,1])$ such that $f_{i}$ is identically 1 on $\bar{U}_{i}$ and identically zero on $M \backslash V_{i}$ and put $f_{k+1}:=1-\sum_{i} f_{i}$.

Now fix arbitrary sections $s_{i} \in \Gamma_{c}\left(E_{i} M\right)$. For each multiindex $I=\left(i_{1}, \ldots, i_{k}\right)$ of integers $i_{1}, \ldots, i_{k}$ between 1 and $k+1$ there is an open subset of $M$ on which all the sections $f_{i_{1}} s_{1}, \ldots, f_{i_{k}} s_{k}$ vanish by the choice of the functions. So by the previous lemma we get an absolutely invariant element $D\left(f_{i_{1}} s_{1}, \ldots, f_{i_{k}} s_{k}\right)_{\text {inv }} \in \mathcal{F}(M)$. Let us define

$$
\lambda\left(s_{1}, \ldots, s_{k}\right)=\lambda^{f_{1}, \ldots, f_{k}}\left(s_{1}, \ldots, s_{k}\right)=\sum_{I} D\left(f_{i_{1}} s_{1}, \ldots, f_{i_{k}} s_{k}\right)_{\mathrm{inv}} .
$$

This operator is obviously $k$-linear, and since the presheaf $\mathcal{F}$ is admissible it is continuous provided that $D$ is continuous.

2.7. Lemma. The operator $\lambda$ does not depend on the choices of $U_{i}, V_{i}$ and $f_{i}$. Moreover if for some open subset $U$ of $M$ the sections $s_{j} \in \Gamma_{c}\left(E_{j} M\right)$ all restrict to zero on $U$, then $r_{U}^{M}\left(\lambda\left(s_{1}, \ldots, s_{k}\right)\right)=r_{U}^{M}\left(D\left(s_{1}, \ldots, s_{k}\right)\right)$.

Proof. We show that we may replace $\left(U_{j}, V_{j}, f_{j}\right)$ by $\left(U_{j}^{\prime}, V_{j}^{\prime}, f_{j}^{\prime}\right)$ for one fixed $j$ if the new set of triples still satisfies the conditions of 2.6. Clearly, it suffices to do this for $j=1$. So let us take a second choice $U_{i}^{\prime}, V_{i}^{\prime}$ and $f_{i}^{\prime}$ where the data do not change for $i>1$.

By the multilinearity of $D$, we get

$$
\begin{aligned}
& D\left(f_{i_{1}} s_{1}, \ldots, f_{i_{k}} s_{k}\right)-D\left(f_{i_{1}}^{\prime} s_{1}, \ldots, f_{i_{k}}^{\prime} s_{k}\right)= \\
& \quad=\sum_{j} D\left(f_{i_{1}}^{\prime} s_{1}, \ldots, f_{i_{j-1}}^{\prime} s_{j-1},\left(f_{i_{j}}-f_{i_{j}}^{\prime}\right) s_{j}, f_{i_{j+1}} s_{j+1}, \ldots, f_{i_{k}} s_{k}\right) .
\end{aligned}
$$

By the assumption on $\bar{V}_{1}^{\prime}$ all sections occurring as arguments in this equation vanish simultaneously on some open subset in $M$, so we get the analogous equation for the invariant elements $D\left(f_{i_{1}} s_{1}, \ldots, f_{i_{k}} s_{k}\right)_{\text {inv }}-D\left(f_{i_{1}}^{\prime} s_{1}, \ldots, f_{i_{k}}^{\prime} s_{k}\right)_{\text {inv }}$. Thus we obtain

$$
\begin{aligned}
\lambda^{f_{1}, \ldots, f_{k}} & \left(s_{1}, \ldots, s_{k}\right)-\lambda^{f_{1}^{\prime}, \ldots, f_{k}^{\prime}}\left(s_{1}, \ldots, s_{k}\right)= \\
\quad= & \sum_{j=1}^{k} \sum_{I} D\left(f_{i_{1}}^{\prime} s_{1}, \ldots, f_{i_{j-1}}^{\prime} s_{j-1},\left(f_{i_{j}}-f_{i_{j}}^{\prime}\right) s_{j}, f_{i_{j+1}} s_{j+1}, \ldots, f_{i_{k}} s_{k}\right)_{\mathrm{inv}} .
\end{aligned}
$$

But in fact only the terms with either $i_{j}=1$ or $i_{j}=k+1$ can contribute, since otherwise $f_{i_{j}}=f_{i_{j}}^{\prime}$ and so one argument vanishes globally on $M$. Thus

$$
\begin{aligned}
& \lambda^{f_{1}, \ldots, f_{k}}\left(s_{1}, \ldots, s_{k}\right)-\lambda^{f_{1}^{\prime}, \ldots, f_{k}^{\prime}}\left(s_{1}, \ldots, s_{k}\right)= \\
= & \sum_{j=1}^{k} \sum_{\left(i_{1}, \ldots, i_{j-1}, i_{j+1}, \ldots, i_{k}\right)}\left(D\left(f_{i_{1}}^{\prime} s_{1}, \ldots, f_{i_{j-1}}^{\prime} s_{j-1},\left(f_{1}-f_{1}^{\prime}\right) s_{j}, f_{i_{j+1}} s_{j+1}, \ldots, f_{i_{k}} s_{k}\right)_{\mathrm{inv}}\right. \\
& \left.+D\left(f_{i_{1}}^{\prime} s_{1}, \ldots, f_{i_{j-1}}^{\prime} s_{j-1},\left(f_{k+1}-f_{k+1}^{\prime}\right) s_{j}, f_{i_{j+1}} s_{j+1}, \ldots, f_{i_{k}} s_{k}\right) \text { inv }\right) .
\end{aligned}
$$


Since $f_{k+1}-f_{k+1}^{\prime}=f_{1}^{\prime}-f_{1}$ by definition, the two terms in the most inner sum annihilate each other and so the whole expression vanishes.

Finally if all $s_{j}$ restrict to zero on an open subset $U$ of $M$ then by the first part of this proof we may compute $\lambda$ using triples $\left(U_{i}, V_{i}, f_{i}\right)$ such that $f_{1}, \ldots, f_{k}$ have support contained in $U$. Then by definition $\lambda\left(s_{1}, \ldots, s_{k}\right)=D\left(f_{k+1} s_{1}, \ldots, f_{k+1} s_{k}\right)_{\text {inv }}=$ $D\left(s_{1}, \ldots, s_{k}\right)_{\text {inv }}$ and thus again by definition

$$
r_{U}^{M}\left(\lambda\left(s_{1}, \ldots, s_{k}\right)\right)=r_{U}^{M}\left(D\left(s_{1}, \ldots, s_{k}\right)\right)
$$

2.8. Lemma. The operator $\lambda$ commutes with Lie derivatives, i.e. for any $X \in$ $\mathcal{X}(M)$ and any $s_{i} \in \Gamma_{c}\left(E_{i} M\right)$ we have $\sum_{i=1}^{k} \lambda\left(s_{1}, \ldots, \mathcal{L}_{X} s_{i}, \ldots, s_{k}\right)=0$.

Proof. First we may clearly assume that $X$ has compact support. Thus we may assume that the support of $X$ is arbitrarily small. So we may choose $U_{i}, V_{i}$ and $f_{i}$ as in 2.6 in such a way that the support of $X$ is contained in $U_{1}$. Since $f \mathcal{L}_{X} s=$ $\mathcal{L}_{X}(f s)-(X f) s$ and all $f_{i}$ are constant on the support of $X$, we see that $\mathcal{L}_{X}$ commutes with the multiplication by $f_{i}$ for each $i$ and thus we get

$$
\sum_{j=1}^{k} \lambda\left(s_{1}, \ldots, \mathcal{L}_{X} s_{j}, \ldots, s_{k}\right)=\sum_{j=1}^{k} \sum_{I} D\left(f_{i_{1}} s_{1}, \ldots, \mathcal{L}_{X} f_{i_{j}} s_{j}, \ldots, f_{i_{k}} s_{k}\right)_{\mathrm{inv}} .
$$

Now we exchange the two sums. For a fixed $I$ all sections $f_{i_{\ell}} s_{\ell}$ and $\mathcal{L}_{X} f_{i_{\ell}} s_{\ell}$ vanish simultaneously on some open subset. Then for the restrictions to this subset we get

$$
\begin{aligned}
\sum_{j=1}^{k} D( & \left.f_{i_{1}} s_{1}, \ldots, \mathcal{L}_{X} f_{i_{j}} s_{j}, \ldots, f_{i_{k}} s_{k}\right)_{\mathrm{inv}}= \\
& =\sum_{j=1}^{k} D\left(f_{i_{1}} s_{1}, \ldots, \mathcal{L}_{X} f_{i_{j}} s_{j}, \ldots, f_{i_{k}} s_{k}\right)=\mathcal{L}_{X}\left(D\left(f_{i_{1}} s_{1}, \ldots, f_{i_{k}} s_{k}\right)\right)=0
\end{aligned}
$$

since the restriction of $D\left(f_{i_{1}} s_{1}, \ldots, f_{i_{k}} s_{k}\right)$ to this subset is absolutely invariant. Since absolutely invariant elements are determined by their restrictions to any open subset the sum vanishes globally and the result follows.

2.9. Theorem. Let $E_{1}, \ldots, E_{k}$ be vector bundle functors defined on the category $\mathcal{M} f_{m}^{+}, m \geq 2$, and let $\mathcal{F}$ be an admissible natural presheaf over a connected $m-$ dimensional manifold $M$. Let $D: \Gamma_{c}\left(E_{1} M\right) \times \ldots \times \Gamma_{c}\left(E_{k} M\right) \rightarrow \mathcal{F}(M)$ be a k-linear operator which commutes with Lie derivatives. Let $\lambda: \Gamma_{c}\left(E_{1} M\right) \times \ldots \times \Gamma_{c}\left(E_{k} M\right) \rightarrow$ $\mathcal{F}(M)$ be the $k$-linear operator constructed above, and put $\tilde{D}=D-\lambda$. Then we have:

(1) Both $\tilde{D}$ and $\lambda$ commute with Lie derivatives.

(2) If $D$ is separately continuous then so are $\tilde{D}$ and $\lambda$.

(3) $\lambda$ has values in the subspace of absolutely invariant elements.

(4) Suppose that D satisfies the following condition:

Let $U \subset M$ be an open subset, $s_{\ell} \in \Gamma_{c}\left(E_{\ell} M\right)$ for $\ell=1, \ldots, k$. If there are $i$ and $j$ between 1 and $k$ such that $s_{i}$ vanishes on $U$ and the support of $s_{j}$ is contained in $U$, then $D\left(s_{1}, \ldots, s_{k}\right)$ restricts to zero on $U$.

Then $\lambda$ is weakly local and $\tilde{D}$ is local.

The only point that remains to be proved is (4). First we need a lemma: 
2.10. Lemma. Suppose that $D: \Gamma_{c}\left(E_{1} M\right) \times \ldots \times \Gamma_{c}\left(E_{k} M\right) \rightarrow \mathcal{F}(M)$ satisfies the condition of 2.9.(4), and suppose that $s_{1}, \ldots, s_{k}$ are sections such that for some $\ell \leq k$ and some $i_{1}, \ldots, i_{\ell}$ between 1 and $k$ we have $\operatorname{supp}\left(s_{i_{1}}\right) \cap \cdots \cap \operatorname{supp}\left(s_{i_{\ell}}\right)=\emptyset$. Then $D\left(s_{1}, \ldots, s_{k}\right)$ restricts to zero on $M \backslash\left(\cup_{j=1}^{\ell} \operatorname{supp} s_{i_{j}}\right)$.

Proof. We proceed by induction on $\ell$. For $\ell=1$ there is nothing to prove. If $\ell=2$ we assume without loss of generality that $s_{1}$ and $s_{2}$ have disjoint supports. Then $s_{1}$ vanishes on the open set $M \backslash \operatorname{supp}\left(s_{1}\right)$ and $s_{2}$ has support contained in this open set so by the condition 2.9.(4), $D\left(s_{1}, \ldots, s_{k}\right)$ restricts to zero on $M \backslash \operatorname{supp}\left(s_{1}\right)$ and thus also on $M \backslash\left(\operatorname{supp}\left(s_{1}\right) \cup \operatorname{supp}\left(s_{2}\right)\right)$.

So let us assume that $\ell>2$ and that the result has been proved for all integers smaller than $\ell$. Without loss of generality we assume that $\operatorname{supp}\left(s_{1}\right) \cap \cdots \cap \operatorname{supp}\left(s_{\ell}\right)=$ $\emptyset$ and that all intersections of $\ell-1$ of these sets are nonempty. Then for $i=$ $1, \ldots, \ell$ we can construct open sets $U_{i}$ such that $U_{i}$ is an open neighborhood of $\operatorname{supp}\left(s_{1}\right) \cap \cdots \cap \operatorname{supp}\left(s_{i-1}\right) \cap \operatorname{supp}\left(s_{i+1}\right) \cap \cdots \cap \operatorname{supp}\left(s_{\ell}\right)$, the sets $\bar{U}_{i}$ are pairwise disjoint and $\bar{U}_{i} \cap \operatorname{supp}\left(s_{i}\right)=\emptyset$. Next for any $i$ choose a smooth function $f_{i} \in$ $C^{\infty}(M,[0,1])$ such that $f_{i}$ has support contained in $U_{i}$ and is identically one on $\operatorname{supp}\left(s_{1}\right) \cap \cdots \cap \operatorname{supp}\left(s_{i-1}\right) \cap \operatorname{supp}\left(s_{i+1}\right) \cap \cdots \cap \operatorname{supp}\left(s_{\ell}\right)$, and put $f_{\ell+1}=1-$ $\sum_{i=1}^{\ell} f_{i}$. By multilinearity of $D$ we get $D\left(s_{1}, \ldots, s_{k}\right)=\sum_{I} D\left(f_{i_{1}} s_{1}, \ldots, f_{i_{k}} s_{k}\right)$, where $I=\left(i_{1}, \ldots, i_{k}\right)$ runs over all $k$-tuples of integers between 1 and $\ell+1$. Now put $W:=M \backslash\left(\cup_{i=1}^{\ell} \operatorname{supp}\left(s_{i}\right)\right)$. If there are at least two numbers between 1 and $\ell$ in the multiindex $I$, then the two corresponding functions have disjoint supports and thus from above we see that the summand corresponding to $I$ vanishes on an open set which certainly contains $W$. So only summands corresponding to multiindices in which there is only one fixed $j \leq \ell$ can contribute to the restriction to $W$. But if $i_{j}=j$ then by construction $f_{i_{j}} s_{j}=0$ and if $i_{r}=j$ for some $r \neq j$ then the corresponding function has support disjoint to the support of $s_{j}$ and so again the corresponding summand vanishes on an open set which contains $W$. Thus we see that $r_{W}^{M}\left(D\left(s_{1}, \ldots, s_{k}\right)\right)=r_{W}^{M}\left(D\left(f_{\ell+1} s_{1}, \ldots, f_{\ell+1} s_{k}\right)\right)$. But by construction the support of $f_{\ell+1}$ is disjoint to $\operatorname{supp}\left(s_{1}\right) \cap \cdots \cap \operatorname{supp}\left(s_{\ell-1}\right)$, so we see that $\operatorname{supp}\left(f_{\ell+1} s_{1}\right) \cap \cdots \cap \operatorname{supp}\left(f_{\ell+1} s_{\ell-1}\right)=\emptyset$, so by the induction hypothesis $D\left(f_{\ell+1} s_{1}, \ldots, f_{\ell+1} s_{k}\right)$ vanishes on an open set which contains $W$.

2.11. Next we prove that under the condition 2.9.(4) the operator $\lambda$ is weakly local. So assume that $s_{1}, \ldots, s_{k}$ are sections such that $\operatorname{supp}\left(s_{1}\right) \cap \cdots \cap \operatorname{supp}\left(s_{k}\right)=$ $\emptyset$. Choose any triples $\left(U_{i}, V_{i}, f_{i}\right)$ as in 2.6. Then by 2.10 for any multiindex $I=\left(i_{1}, \ldots, i_{k}\right)$ the element $D\left(f_{i_{1}} s_{1}, \ldots, f_{i_{k}} s_{k}\right)$ restricts to zero on the open subset $M \backslash\left(\cup_{j} \operatorname{supp}\left(f_{i_{j}} s_{j}\right)\right)$. By construction this set is nonempty and moreover by definition the restrictions of $D\left(f_{i_{1}} s_{1}, \ldots, f_{i_{k}} s_{k}\right)$ and $D\left(f_{i_{1}} s_{1}, \ldots, f_{i_{k}} s_{k}\right)_{\text {inv }}$ to this subset coincide. Thus $D\left(f_{i_{1}} s_{1}, \ldots, f_{i_{k}} s_{k}\right)_{\text {inv }}=0$ since absolutely invariant elements are determined by their restrictions to arbitrary open sets, and consequently $\lambda\left(s_{1}, \ldots, s_{k}\right)=0$.

2.12. So it remains to show that under the condition 2.9.(4) the operator $\tilde{D}$ is local. So assume that we have given sections $s_{1}, \ldots, s_{k}$ and an open subset $W$ such that $s_{1}$ restricts to zero on $W$. Then we have to show that $r_{W}^{M}\left(D\left(s_{1}, \ldots, s_{k}\right)\right)=$ $r_{W}^{M}\left(\lambda\left(s_{1}, \ldots, s_{k}\right)\right)$. Let $U \subset W$ be open and such that $\bar{U} \subset W$. Put $U_{1}:=U$, $V_{1}:=W$ and choose a function $f_{1}$ and triples $\left(U_{i}, V_{i}, f_{i}\right)$ for $i \geq 2$ like in 2.6. Consider a multiindex $I=\left(i_{1}, \ldots, i_{k}\right)$. If $i_{1}=1$ then $f_{i_{1}} s_{1}=0$ and if $i_{j}=1$ for some other $j$ then the section $f_{i_{j}} s_{j}$ has support contained in $W$, while $f_{i_{1}} s_{1}$ vanishes 
on $W$. So by the proof of 2.10 and multilinearity we see that $r_{W}^{M}\left(D\left(s_{1}, \ldots, s_{k}\right)\right)=$ $\sum_{I: i_{j} \neq 1} r_{W}^{M}\left(D\left(f_{i_{1}} s_{1}, \ldots, f_{i_{k}} s_{k}\right)\right)$.

Next $\lambda\left(s_{1}, \ldots, s_{k}\right)=\sum_{I: i_{j} \neq 1} D\left(f_{i_{1}} s_{1}, \ldots, f_{i_{k}} s_{k}\right)_{\text {inv }}$ by weak locality and multilinearity. For any multiindex $I$ with all $i_{\ell} \neq 1$ the sections $f_{i_{1}} s_{1}, \ldots, f_{i_{k}} s_{k}$ vanish simultaneously on the set $U_{1}=U$. Consequently we see that $r_{U}^{M}\left(\lambda\left(s_{1}, \ldots, s_{k}\right)\right)=$ $r_{U}^{M}\left(D\left(s_{1}, \ldots, s_{k}\right)\right)$.

Now the set $W$ can be covered by countably many open subsets $W_{i}$ such that there are homeomorphisms $w_{i}: W_{i} \rightarrow \mathbb{R}^{m}$. For any $(i, j) \in \mathbb{N}^{2}$ let $W_{i j}$ be the preimage under $w_{i}$ of the open ball of radius $j$ around the origin. Then for all $i, j$ the closure of $W_{i j}$ is contained in $W$. Next take a bijection $\kappa: \mathbb{N} \rightarrow \mathbb{N}^{2}$ and define $U_{\ell}:=\cup_{i \leq \ell} W_{\kappa(i)}$. Then clearly $U_{\ell} \subset U_{\ell+1}$ for all $\ell$ and $\bar{U}_{\ell} \subset W$ and $W=\cup_{\ell \in \mathbb{N}} U_{\ell}$.

From above we see that $\tilde{D}\left(s_{1}, \ldots, s_{k}\right)$ restricts to zero on each set $U_{\ell}$. Since the presheaf $\mathcal{F}$ is admissible we see that $\tilde{D}\left(s_{1}, \ldots, s_{k}\right)$ restricts to zero on $W$ by condition (i) of 2.1.(3).

This finishes the proof of theorem 2.9.

\section{WEAKLY LOCAL NATURAL FUNCTIONALS}

In this section we give a complete description of weakly local multilinear functionals which commute with Lie derivatives. This classification can be found in [Kirillov], but the proofs are only briefly sketched there. To have this paper self contained we present full proofs here. Moreover we weaken some continuity assumptions. The proofs are rather technical, so readers who are not interested in details should only read lemma 3.3 and skip the rest of this section.

3.1. Lemma. Let $\tau: C_{c}^{\infty}\left(\mathbb{R}^{m}, \mathbb{R}\right) \rightarrow \mathbb{R}$ be a not necessarily continuous linear map such that for any $f \in C_{c}^{\infty}\left(\mathbb{R}^{m}, \mathbb{R}\right)$ and any $i=1, \ldots, m$ we have $\tau\left(\partial_{i} f\right)=0$, where $\partial_{i} f$ denotes the $i$-th partial derivative of $f$. Then $\tau(f)=c \int_{\mathbb{R}^{m}} f(x) d x$ for some real constant $c$. In particular $\tau$ is automatically continuous.

Proof. A similar proof to that used for distributions in [Hörmander, 3.1.4 and 3.1.4'] applies. Let us first assume that $m=1$. Choose a function $\psi \in C_{c}^{\infty}(\mathbb{R}, \mathbb{R})$ such that $\int_{\mathbb{R}} \psi=1$. Then for any $f \in C_{c}^{\infty}(\mathbb{R}, \mathbb{R})$ we get

$$
f(t)-\psi(t) \int_{\mathbb{R}} f=\frac{d}{d t} \int_{-\infty}^{t}\left(f(x)-\psi(x) \int_{\mathbb{R}} f\right) d x,
$$

and since the integral over the left hand side obviously vanishes, the function which is differentiated on the right hand side has compact support. Thus we get $0=$ $\tau\left(f-\psi \int_{\mathbb{R}} f\right)$ and hence $\tau(f)=\tau(\psi) \int_{\mathbb{R}} f$.

Next for $m>1$ take the function $\psi$ from above, and for $g \in C_{c}^{\infty}\left(\mathbb{R}^{m-1}, \mathbb{R}\right)$ define $g_{m} \in C_{c}^{\infty}\left(\mathbb{R}^{m}, \mathbb{R}\right)$ by $g_{m}\left(x_{1}, \ldots, x_{m}\right):=g\left(x_{1}, \ldots, x_{m-1}\right) \cdot \psi\left(x_{m}\right)$. Then define $\tau_{m}: C_{c}^{\infty}\left(\mathbb{R}^{m-1}, \mathbb{R}\right) \rightarrow \mathbb{R}$ by $\tau_{m}(g)=\tau\left(g_{m}\right)$. For $f \in C_{c}^{\infty}\left(\mathbb{R}^{m}, \mathbb{R}\right)$ consider $I_{m}(f) \in$ $C_{c}^{\infty}\left(\mathbb{R}^{m-1}, \mathbb{R}\right)$ defined by $I_{m}(f)\left(x_{1}, \ldots, x_{m-1}\right):=\int_{\mathbb{R}} f\left(x_{1}, \ldots, x_{m}\right) d x_{m}$. Now as above one shows that the function $f\left(x_{1}, \ldots, x_{m}\right)-I_{m}(f)\left(x_{1}, \ldots, x_{m-1}\right) \cdot \psi\left(x_{m}\right)$ is the $m$-th partial derivative of a compactly supported smooth function so we see that $\tau(f)=\tau\left(\left(I_{m}(f)\right)_{m}\right)=\tau_{m}\left(I_{m}(f)\right)$. Since by construction $\tau_{m}$ is again linear an vanishes on partial derivatives the result follows by induction. 
3.2. Lemma. Let $\lambda:\left(C_{c}^{\infty}\left(\mathbb{R}^{m}, \mathbb{R}\right)\right)^{k} \rightarrow \mathbb{R}$ be a $k$-linear functional of the form

$$
\lambda\left(f_{1}, \ldots, f_{k}\right)=\sum_{\alpha^{1}, \ldots, \alpha^{k}} \int_{\mathbb{R}^{m}} c_{\alpha^{1} \ldots \alpha^{k}}(x) \partial^{\alpha^{1}} f_{1}(x) \ldots \partial^{\alpha^{k}} f_{k}(x) d x
$$

where each $c_{\alpha^{1} \ldots \alpha^{k}}$ is a continuous function on $\mathbb{R}^{m}$ and the sum is over finitely many $k$-tuples of multiindices $\alpha^{j}=\left(\alpha_{1}^{j}, \ldots, \alpha_{m}^{j}\right)$ of nonnegative integers, and $\partial^{\alpha^{j}}$ is the composition of partial derivatives corresponding to $\alpha^{j}$. If $\lambda$ commutes with partial derivatives, i.e. $\sum_{i=1}^{k} \lambda\left(f_{1}, \ldots, \partial_{\ell} f_{i}, \ldots, f_{k}\right)=0$ for all functions $f_{j} \in C_{c}^{\infty}\left(\mathbb{R}^{m}, \mathbb{R}\right)$ and any $\ell=1, \ldots, m$, then there are constants $d_{\beta^{1}} \ldots \beta^{k-1}$ such that

$$
\lambda\left(f_{1}, \ldots, f_{k}\right)=\sum_{\beta^{1}, \ldots, \beta^{k-1}} \int_{\mathbb{R}^{m}} d_{\beta^{1} \ldots \beta^{k-1}} f_{k}(x) \partial^{\beta^{1}} f_{1}(x) \ldots \partial^{\beta^{k-1}} f_{k-1}(x) d x,
$$

where again the sum is over a finite set of multiindices.

Proof. In order to bring a given expression for $\lambda$ to the required final form we will rewrite its summands recursively. Therefore we need an appropriate ordering on the sets of multi-multi indices. What we will need is $(k-1)$-tuples $\left(\alpha^{1}, \ldots, \alpha^{k-1}\right)$ where each $\alpha^{j}=\left(\alpha_{1}^{j}, \ldots, \alpha_{m}^{j}\right)$ is an $m$-tuple of nonnegative integers. First we order these $m$-tuples by their total degree $\left|\alpha^{j}\right|=\sum \alpha_{i}^{j}$ and within each total degree lexicographically. Then we order the $(k-1)$-tuples by their total degree $\left|\left(\alpha^{1}, \ldots, \alpha^{k-1}\right)\right|=\sum\left|\alpha^{j}\right|$ and within each total degree lexicographically.

So let assume that for some fixed $(k-1)$-tuple $\left(\beta^{1}, \ldots, \beta^{k-1}\right)$ and fixed $m$-tuple $\beta$ we already have an expression for $\lambda$ of the following form:

$$
\begin{aligned}
\lambda\left(f_{1}, \ldots, f_{k}\right) & =\sum_{\left(\alpha^{1}, \ldots, \alpha^{k-1}\right)<\left(\beta^{1}, \ldots, \beta^{k-1}\right)} \int_{\mathbb{R}^{m}} d_{\alpha^{1} \ldots \alpha^{k-1}} f_{k}(x) \partial^{\alpha^{1}} f_{1}(x) \ldots \partial^{\alpha^{k-1}} f_{k-1}(x) d x+ \\
& +\sum_{\beta^{k} \leq \beta} \int_{\mathbb{R}^{m}} c_{\beta^{1} \ldots \beta^{k}}(x) \partial^{\beta^{1}} f_{1}(x) \ldots \partial^{\beta^{k}} f_{k}(x) d x+ \\
& +\sum_{\left(\alpha^{1}, \ldots, \alpha^{k-1}\right)>\left(\beta^{1}, \ldots, \beta^{k-1}\right)} \sum_{\alpha^{k}} \int_{\mathbb{R}^{m}} c_{\alpha^{1} \ldots \alpha^{k}}(x) \partial^{\alpha^{1}} f_{1}(x) \ldots \partial^{\alpha^{k}} f_{k}(x) d x,
\end{aligned}
$$

where the $d$ 's are constants, the c's are continuous functions and $c_{\beta^{1} \ldots \beta^{k-1} \beta}$ is nonzero.

Now fix a relatively compact open subset $U \subset \mathbb{R}^{m}$. Then choose a compactly supported smooth function $f$ on $\mathbb{R}^{m}$ such that $f$ is identically one on $U$, and consider the distribution $f_{k} \mapsto \lambda\left(x^{\beta^{1}} f, \ldots, x^{\beta^{k-1}} f, f_{k}\right)$ on $C_{c}^{\infty}(U, \mathbb{R})$. Then this is given by

$$
\begin{gathered}
f_{k} \mapsto \sum_{\substack{\left(\alpha^{1}, \ldots, \alpha^{k-1}\right)<\left(\beta^{1}, \ldots, \beta^{k-1}\right) \\
\alpha^{i} \leq \beta^{i}}} \int_{\mathbb{R}^{m}} d_{\alpha^{1} \ldots \alpha^{k-1}} \frac{\beta^{1} !}{\left(\beta^{1}-\alpha^{1}\right) !} \cdots \frac{\beta^{k-1} !}{\left(\beta^{k-1}-\alpha^{k-1}\right) !} f_{k}(x) . \\
\cdot x^{\beta^{1}-\alpha^{1}} \ldots x^{\beta^{k-1}-\alpha^{k-1}} d x+ \\
+\sum_{\beta^{k} \leq \beta} \int_{\mathbb{R}^{m}} \beta^{1} ! \ldots \beta^{k} ! c_{\beta^{1} \ldots \beta^{k}}(x) \partial^{\beta^{k}} f_{k}(x) d x
\end{gathered}
$$


where the first sum collects all summands with $\left(\alpha^{1}, \ldots, \alpha^{k-1}\right)<\left(\beta^{1}, \ldots, \beta^{k-1}\right)$, since the remaining terms vanish. Using the commutation of $\lambda$ with partial derivatives and (1) we get:

$$
\begin{array}{r}
\lambda\left(x^{\beta^{1}} f, \ldots, x^{\beta^{k-1}} f, \partial_{i} f_{k}\right)=-\sum_{j=1}^{k-1} \lambda\left(x^{\beta^{1}} f, \ldots, \partial_{i}\left(x^{\beta^{j}} f\right), \ldots, x^{\beta^{k-1}} f, f_{k}\right)= \\
=-\sum_{j} \sum_{\substack{\left(\alpha^{1}, \ldots, \alpha^{k-1}\right)<\left(\beta^{1}, \ldots, \beta^{k-1}\right) \\
\alpha^{i} \leq \beta^{i}}} \int_{\mathbb{R}^{m}} d_{\alpha^{1} \ldots \alpha^{k-1}} \frac{\beta^{1} !}{\left(\beta^{1}-\alpha^{1}\right) !} \cdots \frac{\beta^{k-1} !}{\left(\beta^{k-1}-\alpha^{k-1}\right) !} f_{k}(x) . \\
\cdot x^{\beta^{1}-\alpha^{1}} \ldots \partial_{i}\left(x^{\beta^{j}-\alpha^{j}}\right) \ldots x^{\beta^{k-1}-\alpha^{k-1}} d x+0 .
\end{array}
$$

On the other hand using (2) we compute:

$$
\begin{gathered}
\lambda\left(x^{\beta^{1}} f, \ldots, x^{\beta^{k-1}} f, \partial_{i} f_{k}\right)= \\
\sum_{\substack{\left(\alpha^{1}, \ldots, \alpha^{k-1}\right)<\left(\beta^{1}, \ldots, \beta^{k-1}\right) \\
\alpha^{i} \leq \beta^{i}}} \int_{\mathbb{R}^{m}} d_{\alpha^{1} \ldots \alpha^{k-1}} \frac{\beta^{1} !}{\left(\beta^{1}-\alpha^{1}\right) !} \cdots \frac{\beta^{k-1} !}{\left(\beta^{k-1}-\alpha^{k-1}\right) !} \partial_{i} f_{k}(x) . \\
\cdot x^{\beta^{1}-\alpha^{1}} \ldots x^{\beta^{k-1}-\alpha^{k-1}} d x+ \\
+\sum_{\beta^{k} \leq \beta} \int_{\mathbb{R}^{m}} \beta^{1} ! \ldots \beta^{k} ! c_{\beta^{1} \ldots \beta^{k}}(x) \partial_{i} \partial^{\beta^{k}} f_{k}(x) d x .
\end{gathered}
$$

Now all terms in the first sum of (4) can be integrated by parts and this gives exactly the sum in (3). Thus the remaining sum must be zero. This means that for the restrictions of the c's to $U$ we get the equation

$$
0=\partial_{i}\left(\sum_{\beta^{k} \leq \beta}(-1)^{\left|\beta^{k}\right|+1} \beta^{1} ! \ldots \beta^{k} ! \partial^{\beta^{k}} c_{\beta^{1} \ldots \beta^{k}}\right),
$$

where the derivatives are interpreted in the distributional sense. Since this equation holds over each relatively compact open subset of $\mathbb{R}^{m}$ it holds on $\mathbb{R}^{m}$.

Assume first that $\beta=(0, \ldots, 0)$. Then (5) implies that $c_{\beta^{1} \ldots \beta^{k-1} \beta}$ is constant on $\mathbb{R}^{m}$ and thus we can rewrite the expression (1) for $\lambda$ with $\left(\beta^{1}, \ldots, \beta^{k-1}\right)$ replaced by some greater $\left(\gamma^{1}, \ldots, \gamma^{k-1}\right)$. Moreover in this procedure we do not increase the maximal total degree occurring in the expression.

Next assume that $\beta>(0, \ldots, 0)$. Then we see from (5) that there is a constant $C$ such that

$$
(-1)^{|\beta|+1} \beta ! \partial^{\beta} c_{\beta^{1} \ldots \beta^{k-1} \beta}=C+\sum_{\beta^{k}<\beta}(-1)^{\left|\beta^{k}\right|} \beta^{k} ! \partial^{\beta^{k}} c_{\beta^{1} \ldots \beta^{k}} .
$$

Thus for arbitrary $f_{1}, \ldots, f_{k} \in C_{c}^{\infty}\left(\mathbb{R}^{m}, \mathbb{R}\right)$ we have

$$
\begin{aligned}
& -\int_{\mathbb{R}^{m}} \beta ! c_{\beta^{1} \ldots \beta^{k-1} \beta}(x) \partial^{\beta}\left(f_{k} \partial^{\beta^{1}} f_{1} \ldots \partial^{\beta^{k-1}} f_{k-1}\right)(x) d x= \\
& =C \int_{\mathbb{R}^{m}} f_{k}(x) \partial^{\beta^{1}} f_{1}(x) \ldots \partial^{\beta^{k-1}} f_{k-1}(x) d x+ \\
& \quad+\sum_{\beta^{k}<\beta} \beta^{k} ! c_{\beta^{1} \ldots \beta^{k}}(x) \partial^{\beta^{k}}\left(f_{k} \partial^{\beta^{1}} f_{1} \ldots \partial^{\beta^{k-1}} f_{k-1}\right)(x) d x
\end{aligned}
$$


Expanding the partial derivatives of products in this identity we see that we can express

$$
\int_{\mathbb{R}^{m}} c_{\beta^{1} \ldots \beta^{k-1} \beta}(x) \partial^{\beta^{1}} f_{1}(x) \ldots \partial^{\beta^{k-1}} f_{k-1}(x) \partial^{\beta} f_{k}(x) d x
$$

as a sum of terms of the form $\int_{\mathbb{R}^{m}} \tilde{c}_{\gamma^{1} \ldots \gamma^{k}}(x) \partial^{\gamma^{1}} f_{1}(x) \ldots \partial^{\gamma^{k}} f_{k}(x) d x$ in which either $\left(\gamma^{1}, \ldots, \gamma^{k-1}\right)>\left(\beta^{1}, \ldots, \beta^{k-1}\right)$ or $\left(\gamma^{1}, \ldots, \gamma^{k-1}\right)=\left(\beta^{1}, \ldots, \beta^{k-1}\right)$ and $\gamma^{k}<\beta$. Moreover we always have $\left|\gamma^{1}\right|+\cdots+\left|\gamma^{k}\right| \leq\left|\beta^{1}\right|+\cdots+\left|\beta^{k-1}\right|+|\beta|$.

Thus finitely many applications of this procedure lead to an expression for $\lambda$ of the required form.

3.3. Lemma. Let $E_{1}, \ldots, E_{k}$ be natural vector bundles defined on $\mathcal{M} f_{m}^{+}$, and let $\lambda: \Gamma_{c}\left(E_{1} M\right) \times \ldots \times \Gamma_{c}\left(E_{k} M\right) \rightarrow \mathbb{R}$ be a weakly local separately continuous $k$-linear map, where $M$ is a connected m-dimensional oriented manifold. Then there is a $(k-1)$-linear local natural operator $D^{\lambda}: E_{1} \times \ldots \times E_{k-1} \rightarrow E_{k}^{*} \otimes \Lambda^{m} T^{*}$, where $E_{k}^{*}$ is the bundle functor which assigns to each manifold $M$ the dual bundle of $E_{k} M$, such that

$$
\lambda\left(s_{1}, \ldots, s_{k}\right)=\int_{M}\left\langle D^{\lambda}\left(s_{1}, \ldots, s_{k-1}\right), s_{k}\right\rangle .
$$

Here $\langle, \quad\rangle$ denotes the canonical pairing

$$
\Gamma_{c}\left(E_{k}^{*} M \otimes \Lambda^{m} T^{*} M\right) \times \Gamma_{c}\left(E_{k} M\right) \rightarrow \Gamma_{c}\left(\Lambda^{m} T^{*} M\right) .
$$

In particular $\lambda$ is automatically jointly continuous.

If $k=1$, i.e. $\lambda$ is linear, this means that there is an absolutely invariant section $\sigma$ of $E_{1}^{*} M \otimes \Lambda^{m} T^{*} M$ such that $\lambda(s)=\int_{M}\langle\sigma, s\rangle$. Moreover in this case the continuity follows from the other assumptions.

Proof. Let us first consider the case $M=\mathbb{R}^{m}$. Up to a natural isomorphism, for each natural bundle $E_{j}$ we have $E_{j} \mathbb{R}^{m}=\mathbb{R}^{m} \times V_{j}$, with the action of the translations $t_{x}: y \mapsto x+y$ given by $t_{x} \times \mathrm{id}_{V_{j}}$. Thus the Lie derivatives with respect to the constant fields are just the partial derivatives of the coordinate functions. (This identification is always achieved by $E_{j} \mathbb{R}^{m} \ni v \mapsto\left(p_{j}(v), E\left(t_{-p_{j}}(v)\right)(v)\right) \in \mathbb{R}^{m} \times V_{j}$.)

Now in any $V_{j}$ choose a fixed basis. Then for any multiindex $\left(i_{1}, \ldots, i_{k}\right)$ with $1 \leq i_{j} \leq \operatorname{dim}\left(V_{j}\right)$ consider the $k$-linear functional $\lambda_{i_{1} \ldots i_{k}}: C^{\infty}\left(\mathbb{R}^{m}, \mathbb{R}\right)^{k} \rightarrow \mathbb{R}$ which gives the coordinate expression of $\lambda$. These functionals are clearly separately continuous, weakly local and they commute with partial derivatives if $\lambda$ has these properties.

Let us first treat the case $k=1$, i.e. $\lambda$ is linear. Then without continuity assumptions we conclude from lemma 3.1 that for each $i_{1}$ there is a constant $c_{i_{1}}$ such that $\lambda_{i_{1}}(f)=c_{i_{1}} \int_{\mathbb{R}^{m}} f(x) d x$. Hence for any section $f=\left(f^{1}, \ldots, f^{n_{1}}\right) \in$ $C_{c}^{\infty}\left(\mathbb{R}^{m}, V_{1}\right)$ we have $\lambda(f)=\int_{\mathbb{R}^{m}} \sum_{i} c_{i} f^{i}(x) d x$. Now we interpret $\left(c_{1}, \ldots, c_{n_{1}}\right) d x$ as a section $\sigma$ of $\mathbb{R}^{m} \times V_{1}^{*} \otimes \Lambda^{m} \mathbb{R}^{m *}=E_{1}^{*} \mathbb{R}^{m} \otimes \Lambda^{m} T^{*} \mathbb{R}^{m}$. Hence we have $\lambda(f)=$ $\int_{\mathbb{R}^{m}}\langle f, \sigma\rangle$. Since any Lie derivative of an $m$-form on $\mathbb{R}^{m}$ is exact we get:

$$
\begin{aligned}
0=\int_{\mathbb{R}^{m}} \mathcal{L}_{X}(\langle\sigma, f\rangle)=\int_{\mathbb{R}^{m}}\left\langle\sigma, \mathcal{L}_{X} f\right\rangle & +\int_{\mathbb{R}^{m}}\left\langle\mathcal{L}_{X} \sigma, f\right\rangle= \\
& =\lambda\left(\mathcal{L}_{X} f\right)+\int_{\mathbb{R}^{m}}\left\langle\mathcal{L}_{X} \sigma, f\right\rangle=\int_{\mathbb{R}^{m}}\left\langle\mathcal{L}_{X} \sigma, f\right\rangle .
\end{aligned}
$$


Since this holds for all $f \in \Gamma_{c}(E M)$ and $X \in \mathcal{X}\left(\mathbb{R}^{m}\right)$ we have $\mathcal{L}_{X} \sigma=0$ and thus $\sigma$ is absolutely invariant.

So let us turn back to the general multilinear case. Applying the Schwartz kernel theorem (c.f. [Hörmander, 5.2.1]) to $\lambda_{i_{1} \ldots i_{k}}$ we see that this functional is given by a distribution on $\left(\mathbb{R}^{m}\right)^{k}$, which has support in the diagonal by the weak locality. Thus it is given (at least locally) as

$$
\lambda_{i_{1} \ldots i_{k}}\left(f_{1}, \ldots, f_{k}\right)=\sum_{\alpha_{1}, \ldots, \alpha_{k}} \int_{\mathbb{R}^{m}} c_{\alpha_{1} \ldots \alpha_{k}}(x) \partial^{\alpha_{1}} f_{1}(x) \ldots \partial^{\alpha_{k}} f_{k}(x) d x
$$

where the $c$ 's are continuous functions and the sum is over some finite number of multiindices $\alpha_{j}$ (c.f. [Hörmander, 5.2.3]).

By lemma 3.2 we can express $\lambda_{i_{1} \ldots i_{k}}$ locally as

$$
\sum_{\beta^{1}, \ldots, \beta^{k-1}} \int_{\mathbb{R}^{m}} d_{\beta^{1} \ldots \beta^{k-1}}^{i_{1} \ldots i_{k}} f_{k}(x) \partial^{\beta^{1}} f_{1}(x) \ldots \partial^{\beta^{k-1}} f_{k-1}(x) d x
$$

Fix an open subset $U$ over which such a representation is valid. Then for smooth sections $f_{j} \in C_{c}^{\infty}\left(U, V_{j}\right)$ we have

$$
\lambda\left(f_{1}, \ldots, f_{k}\right)=\sum_{i_{1}, \ldots, i_{k}} \sum_{\beta^{1}, \ldots, \beta^{k-1}} \int_{\mathbb{R}^{m}} d_{\beta^{1} \ldots \beta^{k-1}}^{i_{1} \ldots i_{k}} f_{k}^{i_{k}}(x) \partial^{\beta^{1}} f_{1}^{i_{1}}(x) \ldots \partial^{\beta^{k-1}} f_{k-1}^{i_{k-1}}(x) d x .
$$

We can now define an operator $D_{U}: C_{c}^{\infty}\left(U, V_{1}\right) \times \ldots \times C_{c}^{\infty}\left(U, V_{k-1}\right) \rightarrow C_{c}^{\infty}\left(U, V_{k}^{*} \otimes\right.$ $\left.\Lambda^{m} \mathbb{R}^{m *}\right)$ by

$$
\left\langle D_{U}\left(f_{1}, \ldots, f_{k-1}\right), f_{k}\right\rangle:=\sum_{\substack{\beta^{1}, \ldots, \beta^{k-1} \\ i_{1}, \ldots, i_{k}}} d_{\beta^{1} \ldots \beta^{k-1}}^{i_{1} \ldots i_{k}} f_{k}^{i_{k}}(x) \partial^{\beta^{1}} f_{1}^{i_{1}}(x) \ldots \partial^{\beta^{k-1}} f_{k-1}^{i_{k-1}}(x) d x .
$$

Clearly this is a well defined local operator and over $U$,

$$
\lambda\left(f_{1}, \ldots, f_{k}\right)=\int_{\mathbb{R}^{m}}\left\langle D_{U}\left(f_{1}, \ldots, f_{k-1}\right), f_{k}\right\rangle,
$$

and $D_{U}$ is uniquely determined by this formula. As in the linear case we compute now:

$$
\begin{aligned}
0 & =\int_{\mathbb{R}^{m}} \mathcal{L}_{X}\left\langle D_{U}\left(f_{1}, \ldots, f_{k-1}\right), f_{k}\right\rangle= \\
& =\int_{\mathbb{R}^{m}}\left\langle\mathcal{L}_{X} D_{U}\left(f_{1}, \ldots, f_{k-1}\right), f_{k}\right\rangle+\int_{\mathbb{R}^{m}}\left\langle D_{U}\left(f_{1}, \ldots, f_{k-1}\right), \mathcal{L}_{X} f_{k}\right\rangle= \\
& =\int_{\mathbb{R}^{m}}\left\langle\mathcal{L}_{X} D_{U}\left(f_{1}, \ldots, f_{k-1}\right), f_{k}\right\rangle+\lambda\left(f_{1}, \ldots, f_{k-1}, \mathcal{L}_{X} f_{k}\right)= \\
& =\int_{\mathbb{R}^{m}}\left\langle\mathcal{L}_{X} D_{U}\left(f_{1}, \ldots, f_{k-1}\right), f_{k}\right\rangle-\sum_{i=1}^{k-1} \lambda\left(f_{1}, \ldots, \mathcal{L}_{X} f_{i}, \ldots, f_{k}\right)= \\
& =\int_{\mathbb{R}^{m}}\left\langle\mathcal{L}_{X} D_{U}\left(f_{1}, \ldots, f_{k-1}\right), f_{k}\right\rangle-\sum_{i=1}^{k-1}\left\langle D_{U}\left(f_{1}, \ldots, \mathcal{L}_{X} f_{i}, \ldots, f_{k-1}\right), f_{k}\right\rangle .
\end{aligned}
$$


Thus $D_{U}$ commutes with Lie derivatives, so it extends uniquely to a natural operator by [Čap-Slovák, 3.2], i.e. there is a unique natural operator $D$ on $\mathcal{M} f_{m}^{+}$such that its value on $U$ is $D_{U}$.

Any point of $\mathbb{R}^{m}$ has a neighborhood on which the construction can be carried out as above. Moreover from the uniqueness of the extension we see that for intersecting open sets the natural operators obtained coincide, and thus $\lambda$ is given on $\mathbb{R}^{m}$ by the formula

$$
\lambda\left(f_{1}, \ldots, f_{k}\right)=\int_{\mathbb{R}^{m}}\left\langle D_{\mathbb{R}^{m}}\left(f_{1}, \ldots, f_{k-1}\right), f_{k}\right\rangle .
$$

Finally take an arbitrary manifold $M$. Choose an oriented atlas $\left(U_{i}, u_{i}\right)$ of $M$ such that any $u_{i}$ is a diffeomorphism onto $\mathbb{R}^{m}$. Then we have the induced isomorphisms $E_{j}\left(u_{i}\right): E_{j} M \supset E_{j} U_{i} \rightarrow E_{j} \mathbb{R}^{m}$. Any section of $E_{j} M$ with support in $U_{i}$ can be written as $u_{i}^{*} f_{j}$ for a compactly supported section $f_{j}$. Now by the first part of this proof there is a unique natural operator $D$ such that

$$
\begin{aligned}
\lambda\left(u_{i}^{*} f_{1}, \ldots, u_{i}^{*} f_{k}\right) & =\int_{\mathbb{R}^{m}}\left\langle D_{\mathbb{R}^{m}}\left(f_{1}, \ldots, f_{k-1}\right), f_{k}\right\rangle= \\
& =\int_{\mathbb{R}^{m}}\left\langle\left(u_{i}^{-1}\right)^{*} D_{M}\left(u_{i}^{*} f_{1}, \ldots, u_{i}^{*} f_{k-1}\right), f_{k}\right\rangle= \\
& =\int_{M}\left\langle D_{M}\left(u_{i}^{*} f_{1}, \ldots, u_{i}^{*} f_{k-1}\right), u_{i}^{*} f_{k}\right\rangle .
\end{aligned}
$$

As before the uniqueness implies that the operators obtained from intersecting charts coincide, and since $M$ is connected and any compactly supported section can be written as a finite sum of sections having support in some chart $U_{i}$ the proof is finished.

\section{THE LINEAR CASE}

Now we give a complete description of linear operators between sections of natural vector bundles over oriented manifolds which commute with Lie derivatives. In the next section this result will be used as the starting point for an induction procedure.

4.1. Proposition. Let $F$ be a natural vector bundle defined on the category $\mathcal{M} f_{m}^{+}$ of oriented $m$-dimensional manifolds and orientation preserving local diffeomorphisms and let $M$ be such a manifold which is connected. Then the sheaf of smooth sections of FM is an admissible natural presheaf over $M$.

Proof. By the general theory mentioned in 1.2, there is the canonically defined continuous action of the sheaf of Lie algebras of vector fields on the base manifold on the sections of the natural vector bundle.

Since the sections of a vector bundle form a sheaf, condition (i) of 2.1.(3) is trivially satisfied. On the other hand the existence and uniqueness condition in (ii) of 2.1.(3) follows immediately from 1.3. Finally continuity of the extension operators is trivial since the absolutely invariant sections always form a finite dimensional space. 
4.2. Let $E$ and $F$ be natural vector bundles over $\mathcal{M} f_{m}^{+}$, let $M$ be a connected oriented manifold of dimension $m \geq 2$ and let $D: \Gamma_{c}(E M) \rightarrow \Gamma(F M)$ be a linear operator which commutes with Lie derivatives. Then the condition of 2.9.(4) is automatically satisfied since in the linear case it just means that $D(0)=0$. Thus we may apply theorem 2.9 to split $D$ into a local linear operator $\tilde{D}$ and a linear map $\lambda$ with values in the space of absolutely invariant sections which vanishes on Lie derivatives. Theorem 3.2 of [Čap-Slovák] shows that $\tilde{D}$ is a natural operator, which in particular implies that it is continuous.

To discuss the linear map $\lambda$ let $v_{1}, \ldots, v_{n}$ be a basis of the space of absolutely invariant sections of $F M$ and let $\left\{v_{i}^{*}\right\}$ be the dual basis. Then for any $i=1, \ldots, n$ the map $\lambda^{i}:=v_{i}^{*} \circ \lambda: \Gamma_{c}(E M) \rightarrow \mathbb{R}$ is a linear functional which vanishes on Lie derivatives. Using lemma 3.3 we get:

4.3. Theorem. Let $M$ be a connected oriented smooth manifold of dimension $m \geq 2, E$ and $F$ natural vector bundles defined over $\mathcal{M} f_{m}^{+}, v_{1}, \ldots, v_{n}$ a basis for the space of absolutely invariant sections of $F M$ and let $D: \Gamma_{c}(E M) \rightarrow \Gamma(F M)$ be a linear operator which commutes with Lie derivatives. Then there is a (local) natural operator $\tilde{D}: \Gamma(E M) \rightarrow \Gamma(F M)$ and there are absolutely invariant sections $\sigma_{1}, \ldots, \sigma_{n} \in \Gamma\left(E^{*} M \otimes \Lambda^{m} T^{*} M\right)$ such that

$$
D(s)=\tilde{D}(s)+\sum_{i=1}^{n}\left(\int_{M}\left\langle\sigma_{i}, s\right\rangle\right) v_{i}
$$

So $D$ is an almost natural operator and thus in particular automatically continuous.

4.4. Corollary. In the setting of theorem 4.3 assume that either $F M$ or $E^{*} M \otimes$ $\Lambda^{m} T^{*} M$ has no absolutely invariant sections. Then any linear operator $\Gamma_{c}(E M) \rightarrow$ $\Gamma(F M)$ or $\Gamma(E M) \rightarrow \Gamma(F M)$ which commutes with Lie derivatives is a local natural operator.

Note that the conditions in the corollary are satisfied in many concrete situations. In subbundles of tensor bundles for example absolutely invariant sections can only exist if the numbers of covariant and contravariant indices are equal.

4.5. Corollary. In the setting of theorem 4.3 assume that $M$ is not compact. Then any linear operator $\Gamma_{c}(E M) \rightarrow \Gamma_{c}(F M)$ as well as any continuous linear operator $\Gamma(E M) \rightarrow \Gamma(F M)$ which commutes with Lie derivatives is a (local) natural operator.

Proof. In the case of compact supports the result follows immediately from the fact that on a noncompact manifold no nonzero absolutely invariant section can have compact support by 4.1. In the second case we may restrict the operator to an operator $\Gamma_{c}(E M) \rightarrow \Gamma(F M)$. There it splits by theorem 4.3 as $\tilde{D}+\sum_{i} \lambda_{i} v_{i}$, where the $v_{i}$ are a basis for the space of absolutely invariant sections of $F M$. Now the natural operator $\tilde{D}$ extends continuously to $\Gamma(E M)$, and we consider the difference $\lambda$ between this extension and the original operator. On compactly supported sections this difference has the form $\sum_{i} \lambda_{i} v_{i}$, where $\lambda_{i}(s)=\int_{M}\left\langle\sigma_{i}, s\right\rangle$, for an absolutely invariant section $\sigma_{i} \in \Gamma\left(E^{*} M \otimes \Lambda^{m} T^{*} M\right)$. Now assume that some $\sigma_{i}$, say $\sigma_{1}$ is nonzero. Then using a chart construction one easily concludes that for any open subset $U \subset M$ there is a section $s \in \Gamma_{c}(E M)$ with support contained in $U$ such that $\lambda_{1}(s)=1$. 
Now choose compact subsets $K_{i} \subset M$ such that each $K_{i}$ is contained in the interior of $K_{i+1}$ and such that $M=\cup_{i \in \mathbb{N}} K_{i}$. Then for any $i \in \mathbb{N}$ choose an open subset $U_{i} \subset K_{i} \backslash K_{i-1}$ and a smooth section $s_{i} \in \Gamma_{c}(E M)$ with support contained in $U_{i}$ such that $\lambda_{1}\left(s_{i}\right)=1$. Then any compact subset of $M$ is contained in some $K_{n}$ and thus intersects only finitely many $U_{i}$. Thus the sum $\sum_{i \in \mathbb{N}} s_{i}$ is actually finite over each compact and thus converges in $\Gamma(E M)$. By continuity of $\lambda$ we see that this element is mapped by $\lambda$ to the limit of $\sum_{i=1}^{k} \lambda\left(s_{i}\right)$. But this sum is divergent by construction.

Thus we see that $\lambda$ vanishes on all compactly supported sections and since these are dense in all sections the result follows.

4.6. Corollary. Let $E$ and $F$ be vector bundle functors on $\mathcal{M} f_{m}^{+}$. The natural presheaf $\mathcal{F}(U):=L\left(\Gamma_{c}(E U) ; \Gamma(F U)\right)$ of continuous linear operators is admissible.

Proof. By definition, an element $D \in \mathcal{F}(U):=L\left(\Gamma_{c}(E U) ; \Gamma(F U)\right)$ is absolutely invariant if and only if $D$ commutes with Lie derivatives. But then $D$ is of the form $D(s)=\tilde{D}(s)+\sum_{i}\left(\int_{M}\left\langle\sigma_{i}, s\right\rangle\right) v_{i}$ with $\tilde{D}$ natural and $\sigma_{i}$ absolutely invariant. Thus both summands are completely determined by any restriction to an open submanifold. Moreover using 1.6 and 4.1 we see that the space of absolutely invariant elements is finite dimensional and thus condition (ii) of 2.1.(3) is satisfied.

Let $U \subset M$ be open and let $U_{i}, i \in \mathbb{N}$, be a system of open subsets such that $U_{i} \subset U_{i+1}$ for all $i$ and such that $U=\cup_{i \in \mathbb{N}} U_{i}$. Suppose that $D \in L\left(\Gamma_{c}\left(E_{U}\right) ; \Gamma(F U)\right)$ restricts to zero on each $U_{i}$. Take a section $s \in \Gamma_{c}(E U)$. Then the support of $s$ is contained in some $U_{i}$ and thus in all $U_{j}$ where $j>i$. Hence by assumption $D(s)$ vanishes on each $U_{j}$ and thus on the union of all $U_{j}$ and so condition (i) of 2.1.(3) is satisfied as well.

\section{Proof of Theorem 1.9}

The main idea is to view $k$-linear operators between sections of vector bundles as $\ell$-linear operators with values in the natural presheaf of $(k-\ell)$-linear operators, as defined in example 2.3.(2), to apply theorem 2.9 and to use induction.

5.1. Definition. Let $E_{1}, \ldots, E_{k}, E$ be natural vector bundles defined on $\mathcal{M} f_{m}^{+}$, and let $D: \Gamma_{c}\left(E_{1} M\right) \times \ldots \times \Gamma_{c}\left(E_{k} M\right) \rightarrow \Gamma(E M)$ be a $k$-linear separately continuous operator, where $M$ is an $m$-dimensional oriented manifold. For an $\ell$-tuple $\left(i_{1}, \ldots, i_{\ell}\right)$ of integers with $1 \leq i_{1}<\cdots<i_{\ell} \leq k$ we define the associated $\ell$-linear operator of type $\left(i_{1}, \ldots, i_{\ell}\right)$ to $D$ to be the operator

$D^{i_{1}, \ldots, i_{\ell}}: \Gamma_{c}\left(E_{i_{1}} M\right) \times \ldots \times \Gamma_{c}\left(E_{i_{\ell}} M\right) \rightarrow L\left(\Gamma_{c}\left(E_{i_{\ell+1}} M\right) \times \ldots \times \Gamma_{c}\left(E_{i_{k}} M\right) ; \Gamma(E M)\right)$

given by $D^{i_{1}, \ldots, i_{\ell}}\left(s_{i_{1}}, \ldots, s_{i_{\ell}}\right)\left(s_{i_{\ell+1}}, \ldots, s_{i_{k}}\right):=D\left(s_{1}, \ldots, s_{k}\right)$. Here $\left(i_{\ell+1}, \ldots, i_{k}\right)$ denotes the ordered sequence of integers between 1 and $k$ which do not occur in $\left(i_{1}, \ldots, i_{\ell}\right)$.

5.2. In the notation of 5.1 assume that $D$ commutes with Lie derivatives. Then by definition of the Lie derivatives on the natural presheaf of $(k-\ell)$-linear operators any associated $\ell$-linear operator commutes with Lie derivatives, too.

Next the condition 2.9.(4) can be interpreted nicely in this situation. Consider the operator $D^{i_{1}, \ldots, i_{\ell}}$ from above. Then the condition of 2.9(4) for fixed $j$ means that if any of the other sections $s_{i_{1}}, \ldots, s_{i_{\ell}}$ vanishes on an open subset $U \subset M$ 
which contains the support of $s_{i_{j}}$ then $D^{i_{1}, \ldots, i_{\ell}}\left(s_{i_{1}}, \ldots, s_{i_{\ell}}\right)$ restricts to zero on $U$. This in turn means that if all $s_{i_{\ell+1}}, \ldots, s_{i_{k}}$ have support contained in $U$, then $D\left(s_{1}, \ldots, s_{k}\right)$ restricts to zero on $U$. But this is exactly equivalent to locality of the operator $D^{i_{1}, \ldots, i_{j-1}, i_{j+1}, \ldots, i_{\ell}}$.

Thus the condition in 2.9.(4) means for $\ell$-linear operators with values in the presheaf of $(k-\ell)$-linear operators of the type we consider exactly that all associated $(\ell-1)$-linear operators with values in the presheaves of $(k-\ell+1)$-linear operators are local.

Before we can prove our main theorem we have to study the locality properties of almost natural operators.

5.3. Lemma. Let $D$ be an elementary almost natural $k$-linear operator of the type $\left(I^{1}, \ldots, I^{r}, J\right)$, and let $I$ be any subset of $\{1, \ldots, k\}$. Then the associated operator $D^{I}$ is local if and only if no $I^{j}$ is a subset of $I$.

Proof. Let us assume that no $I^{j}$ is a subset of $I$, take some $i \in I$ and consider a section $s_{i}$ which vanishes on some open subset $U$ of $M$. If $i \in J$ then clearly $D\left(s_{1}, \ldots, s_{k}\right)$ vanishes on $U$ for arbitrary sections $s_{\ell}, \ell \neq i$. On the other hand if $i$ is in some $I^{j}$ then by assumption there is some $\ell \in I^{j}$ which is not in $I$. Then for any section $s_{\ell}$ with support contained in $U$ we have $\lambda^{j}\left(\ldots, s_{i}, \ldots, s_{\ell}, \ldots\right)=0$ by weak locality of $\lambda^{j}$.

Conversely assume that $I^{j} \subset I$. Choose a small open subset $U$ of $M$. Then we can find sections $s_{n}$ for $n \in I^{j}$ which have support in $M \backslash U$ such that $\lambda^{j}$ is nonzero on these sections. On the other hand for all $\ell \neq j$ we can find sections corresponding to $I^{\ell}$ with support contained in $U$ on which $\lambda^{\ell}$ is nonzero. Moreover we can also find sections corresponding to $J$ with support in $U$ on which the local natural part does not vanish, and hence we get a nonzero result over $U$ which contradicts locality of $D^{I}$.

5.4. Next let us consider a general almost natural operator $D$. By definition we can write $D$ as a sum of nonzero elementary almost natural operators.

Lemma. Let $D$ be an almost natural operator and let $I$ be a subset of $\{1, \ldots, k\}$. Then the associated operator $D^{I}$ is local if and only if the associated operator of type I to any of the elementary summands is local.

Moreover, any set of elementary almost natural operators of pairwise different type is linearly independent.

Proof. The non-trivial part of the proof is to show that if $D^{I}$ is local then each elementary summand has the same locality property. By 5.3 this means that the elementary summands must not involve a set $I^{j} \subset I$ in their types. We may assume without loss of generality that if there are several elementary almost natural operators of the same type in this sum, then their local natural parts (corresponding to the last set in the type) are linearly independent. From now on we will assume that all representations of almost natural operators as sums of elementary ones are of this form.

Suppose that $D$ and $I$ are given and take some $i_{0} \in I$ such that there is an elementary summand which has in its type a set $I^{j} \subset I$ which contains $i_{0}$, and consider only those summands which have this property. Next consider only those operators for which the corresponding set $I^{j}$ is of minimal cardinality. Next take the minimal cardinality of the other sets in the types of the remaining operators 
and consider only those which have a set of this cardinality in their type. Next take again the minimal cardinality of the remaining sets and the corresponding operators and so on up to a point where there are only operators left which have all the same cardinalities of sets in their types. Now choose one of the remaining operators and renumber the bundles in such a way that $i_{0}$ becomes 1 and the operator has type $I^{1}=\left\{1,2, \ldots, n_{1}\right\}, \ldots, I^{r}=\left\{n_{1}+\cdots+n_{r-1}+1, \ldots, n_{1}+\cdots+n_{r}\right\}, J=$ $\left\{n_{r}+1, \ldots, k\right\}$, with $n_{2} \leq n_{3} \leq \cdots \leq n_{r}$. Assume that it is of the form $\lambda^{1} \ldots \lambda^{r} \tilde{D}$. Now choose an open subset $U$ of $M$ and sections $s_{1}, \ldots, s_{n_{1}}$ which all vanish on $U$ such that $\lambda^{1}$ is nonzero on these sections. Next choose $r$ disjoint open subsets $U_{2}, \ldots, U_{r+1}$ contained in $U$, for any $i \leq r$ choose sections with support in $U_{i}$ on which $\lambda^{i}$ is nonzero and choose sections with support contained in $U_{r+1}$ on which $\tilde{D}$ is nonzero.

Since all $\lambda^{i}$ are weakly local (cf. 1.7) we see that any operator in the linear combination which does not vanish identically on these sections must belong to those which remained at the end of our choices. So let us consider such operators, and let us denote their types with tildes. By definition of the type we must have $1 \in \tilde{I}^{1}$ or $1 \in \tilde{J}$. By minimality of the cardinality of $I^{1}$ we must then have $\tilde{I}^{1}=I^{1}$ or $\tilde{J}=I^{1}$ to get something nonzero. If $\tilde{J}=I^{1}$ we must have $n_{1}+1 \in \tilde{I}^{1}$ so again by minimality we must have $\tilde{I}^{1}=I^{2}$, then $\tilde{I}^{2}=I^{3}$ and so on. On the other hand if $\tilde{I}^{1}=I^{1}$ then $n_{1}+1 \in \tilde{I}_{2}$ or $n_{1}+1 \in \tilde{J}$ and again the only possibility to get a different type is $\tilde{J}=I^{2}, \tilde{I}^{2}=I^{3}$ and so on. Thus we see that the only operators which can produce something nonzero are those whose types satisfy $\tilde{I}^{j}=I^{j}$ for $j \leq \ell, \tilde{I}^{j}=I^{j+1}$ for $\ell<j \leq r, \tilde{I}^{r}=J$ and $\tilde{J}=I^{\ell+1}$, or those with $\tilde{J}=\emptyset$ for which we must have $\tilde{I}^{j}=I^{j}$ for $j \leq r$ and $\tilde{I}^{r+1}=J$.

If $D^{I}$ is local, these operators must add up to zero on $U$ for each choice of sections as above. The operators with $\tilde{J} \neq \emptyset$ produce section with support in $U_{\ell}$ if $\tilde{J}=I^{\ell}$, while the ones with $\tilde{J}=\emptyset$ produce absolutely invariant sections which in particular have no zeros. Thus for any remaining type the operators of this type have to add up to zero on some $U_{\ell}$ or on $U$. Since we may assume that for each fixed type the occurring local natural parts are linearly independent this implies that the individual elementary summands must already be zero. Thus, altogether, if $D^{I}$ is local, then there is no elementary summand with $I_{j} \subset I$ in its type.

This concludes the proof of the first assertion of the lemma. The linear independence of any set of elementary almost natural operators with pairwise different types can be proved completely analogously. Indeed, if we assume that we can write the zero operator as a linear combination of elementary almost natural operators of different types, then we take the minimal cardinality of all sets occurring in the types of the operators and consider those which have one set of this cardinality in their type, and so on, exactly as above. At the end we choose $r+1$ disjoint open sets $U_{i} \subset M$ and sections supported in the appropriate $U_{i}$. The same arguments as above then apply.

5.5. Corollary. Let $D$ be an almost natural operator on a manifold $M, U$ an open subset of $M$ and $I$ any subset of $\{1, \ldots, k\}$. If the operator $\left(\left.D\right|_{U}\right)^{I}$ associated to the restriction of $D$ to $U$ is local then $D^{I}$ is local.

Proof. This is clear since by 5.3 and 5.4 the locality properties of an almost natural operator are independent of the manifold on which the operator is defined.

5.6. Now we can pass to the proof of our main result, the theorem 1.9: 
Theorem. Let $E_{1}, \ldots, E_{k}, E$ be natural vector bundles defined on the category $\mathcal{M} f_{m}^{+}, m \geq 2$, and let $M$ be a connected oriented smooth manifold of dimension $m$. Then any separately continuous $k$-linear operator $D: \Gamma_{c}\left(E_{1} M\right) \times \ldots \times \Gamma_{c}\left(E_{k} M\right) \rightarrow$ $\Gamma(E M)$ which commutes with Lie derivatives is an almost natural operator. In particular any such operator is automatically jointly continuous and the space of such operators is always finite dimensional and independent of the manifold $M$.

Proof. We proceed by induction on $k$. For $k=1$ the theorem has been proved in section 4. So let us assume that $k \geq 2$ and that the theorem has been proved for all $\ell<k$. Using the induction hypothesis, the space of absolutely invariant elements in the natural presheaf $U \mapsto L\left(\Gamma_{c}\left(E_{i_{1}} U\right), \ldots, \Gamma_{c}\left(E_{i_{\ell}} U\right) ; \Gamma(E U)\right)$ is finite dimensional and each of them is determined by any restriction to an open subset. Thus, the presheaf satisfies condition (ii) of 2.1.(3). Furthermore, exactly as in the proof of 4.6 we verify condition (i) of 2.1.(3). Hence the natural presheaf $U \mapsto L\left(\Gamma_{c}\left(E_{i_{1}} U\right), \ldots, \Gamma_{c}\left(E_{i_{\ell}} U\right) ; \Gamma(E U)\right)$ is admissible for all $\ell<k$. So we may apply theorem 2.9 to the continuous linear operator $D^{1}: \Gamma_{c}\left(E_{1} M\right) \rightarrow$ $L\left(\Gamma_{c}\left(E_{2} M\right), \ldots, \Gamma_{c}\left(E_{k} M\right) ; \Gamma(E M)\right)$. Thus we see that $D^{1}=\tilde{D}^{1}+\lambda^{1}$, where $\tilde{D}^{1}$ is a local operator and $\lambda^{1}$ is a continuous linear functional with values in the space of absolutely invariant elements of $L\left(\Gamma_{c}\left(E_{2} M\right), \ldots, \Gamma_{c}\left(E_{k} M\right) ; \Gamma(E M)\right)$, which are by definition exactly the operators which commute with Lie derivatives. By induction this is the finite dimensional space of almost natural operators, so using proposition 3.3 we see that $\lambda^{1}$ gives an almost natural operator.

So let us assume that $D$ has the property that $D^{1}$ is local. Then consider the associated continuous linear operator $D^{2}$. Again by 2.9 this operator splits as $D^{2}=\tilde{D}^{2}+\lambda^{2}$, where $\tilde{D}^{2}$ is local and $\lambda^{2}$ again gives rise to some almost natural operator. Next assume that $U \neq \emptyset$ is open and that $s_{2} \in \Gamma_{c}\left(E_{2} M\right)$ has support contained in $M \backslash \bar{U}$. Then by lemma 2.7, $r_{U}^{M}\left(\lambda^{2}\left(s_{2}\right)\right)=r_{U}^{M}\left(D^{2}\left(s_{2}\right)\right)$. Then suppose that we take a section $s_{1} \in \Gamma_{c}\left(E_{1} M\right)$ which has support contained in $U$ and vanishes on some open subset $V$ of $U$ and sections $s_{i} \in \Gamma_{c}\left(E_{i} M\right)$ with support contained in $V$ for $i=3, \ldots, k$. Then by construction $\operatorname{supp}\left(s_{i}\right) \subset M \backslash \operatorname{supp}\left(s_{1}\right)$ for all $i>1$, so $D\left(s_{1}, \ldots, s_{k}\right)=D^{2}\left(s_{2}\right)\left(s_{1}, s_{3}, \ldots, s_{k}\right)$ restricts to zero on $M \backslash \operatorname{supp}\left(s_{1}\right)$ and thus we see that $\lambda^{2}\left(s_{2}\right)\left(s_{1}, s_{3}, \ldots, s_{k}\right)$ restricts to zero on $V$. Thus $\lambda^{2}\left(s_{2}\right)$ restricts on $U$ to an almost natural operator which has the property that the associated operator of type 1 is local, so by $5.5 \lambda^{2}\left(s_{2}\right)$ has this property. Since any section $s_{2}$ can be written as the sum of two sections having supports in some open subsets $U \neq M$ this holds for any $s_{2}$. Consequently the $k$-linear operator associated to $\lambda^{2}$ also has the property that its associated operator of type 1 is local and thus the same holds for the $k$-linear operator associated to $\tilde{D}_{2}$.

Together we see that subtracting almost natural operators we come from the original operator $D$ to an operator $\hat{D}$ such that the associated operators $\hat{D}^{1}$ and $\hat{D}^{2}$ are local.

Iterating this procedure we see that subtracting suitable almost natural operators from $D$ we arrive at an operator which has the property that all associated linear operators are local. So let us assume that $D$ itself already has this property.

Then we consider the first associated bilinear operator $D^{1,2}$. By theorem 2.9 this operator splits as $D^{1,2}=\tilde{D}^{1,2}+\lambda^{1,2}$ where $\tilde{D}^{1,2}$ is local and $\lambda^{1,2}$ is weakly local. Using the induction hypothesis and 3.3 we see that $\lambda^{1,2}$ gives again rise to an almost natural operator. Similar arguments as above show that the $k$-linear operator corresponding to $\tilde{D}^{1,2}$ has the property that all associated linear operators 
and the associated bilinear operator of type $(1,2)$ are local. Let us again write $D$ for this operator.

Then we consider the associated operator $D^{1,3}$. By 2.9 this splits as $D^{1,3}=$ $\tilde{D}^{1,3}+\lambda^{1,3}$, with $\tilde{D}^{1,3}$ local and $\lambda^{1,3}$ weakly local, and again $\lambda^{1,3}$ gives rise to

an almost natural operator. Now consider the $k$-linear operator $\tilde{D}$ associated to $\tilde{D}^{1,3}$. We claim that its associated operator of type $(1,2)$ is local. Let us first assume that we have a section $s_{1}$ which vanishes on some open subset $U$ of $M$ an sections $s_{i}$ with support in $U$ for $i \geq 3$. Then in particular $s_{1}$ and $s_{3}$ have disjoint supports and hence $\lambda^{1,3}\left(s_{1}, s_{3}\right)=0$ by weak locality. Consequently for such sections $\tilde{D}$ coincides with $D$ and thus vanishes on $U$. Moreover similarly as before one shows that $\lambda^{1,3}$ has values in the space of almost natural operators which have the property that the associated linear operator corresponding to $s_{2}$ is local. Thus if we have a section $s_{2}$ which vanishes on some open subset $U$ while the sections $s_{i}$ have support in $U$ for all $i \geq 3$ then $\lambda^{1,3}\left(s_{1}, s_{3}\right)\left(s_{2}, s_{4}, \ldots, s_{k}\right)$ restricts to zero on $U$. But since $D^{1,2}$ is local the same is true for $D^{1,3}\left(s_{1}, s_{3}\right)\left(s_{2}, s_{4}, \ldots, s_{k}\right)$ and thus also $\tilde{D}^{1,3}\left(s_{1}, s_{3}\right)\left(s_{2}, s_{4}, \ldots, s_{k}\right)$ restricts to zero on $U$. So again no locality properties are lost.

Iterating this procedure we see that subtracting suitable almost natural operators we arrive at an operator such that all associated bilinear (and thus all associated linear operators) are local. Next we consider the associated trilinear operators in some succession, then the 4-linear ones and so on until we arrive at an operator which has the property that all associated $(k-1)$-linear operators are local. To this operator we can now directly apply theorem 2.9 to split it into a local and a weakly local part which by 3.3 is again an almost natural operator.

Finally from 1.6 it is clear that the space of almost natural $k$-linear operators is again finite dimensional.

\section{Examples and Applications}

In order to illustrate the strength of our results, we shall discuss several concrete geometrical problems. In particular we shall give a completely algebraic characterization of several well known brackets, the Lie bracket, the Schouten bracket, the Schouten-Nijenhuis bracket and a bracket closely related to the Frölicher-Nijenhuis bracket.

6.1. Remark. In fact the only point where we need the continuity assumption on the operators is the description of weakly local multilinear functionals which commute with Lie derivatives. Hence using assumptions which make sure that only linear functionals can occur we can obtain automatic continuity results. We carry this out only for the bilinear case.

6.2. Corollary. Let $E_{1}, E_{2}$ and $F$ be natural vector bundles defined on $\mathcal{M} f_{m}^{+}$such that $F$ has no absolutely invariant sections. Let $M$ be a smooth oriented manifold of dimension $m \geq 2$ and let $D: \Gamma_{c}\left(E_{1} M\right) \times \Gamma_{c}\left(E_{2} M\right) \rightarrow \Gamma(F M)$ be a (not necessarily continuous) bilinear operator which commutes with Lie derivatives. Then $D$ is an almost natural operator and thus in particular jointly continuous.

Proof. Since we proved the description of the linear weakly local functionals commuting with Lie derivatives without continuity assumptions the proof of 4.6 shows also that the presheaf $\mathcal{F}(U):=\tilde{L}\left(\Gamma_{c}\left(E_{i} U\right), \Gamma(F U)\right)$ of not necessarily continuous linear operators is admissible. Now as in the proof of the main theorem subtracting 
almost natural operators from $D$ we arrive at an operator such that both associated linear operators are local. Applying to this operator theorem 2.9 we see that it must be local since $F$ has no absolutely invariant sections.

6.3. The operators on exterior forms. Our first application will give a complete description of all $k$-linear separately continuous operators $D: \Gamma_{c}\left(\Lambda^{p_{1}} T^{*} M\right) \times$ $\ldots \times \Gamma_{c}\left(\Lambda^{p_{k}} T^{*} M\right) \rightarrow \Gamma\left(\Lambda^{q} T^{*} M\right)$ commuting with the Lie derivatives, where $M$ is an arbitrary $m$-dimensional oriented connected manifold, $m \geq 2$.

According to theorem 1.9, D must be an almost natural operator. Thus all operators in question are linearly generated by the possible elementary almost natural operators. So let us focus on the multilinear functionals $\lambda$ and local natural operators $\tilde{D}$ which may appear in $D$. First assume $q=0$ and assume $D=\lambda$ is a multilinear functional vanishing on Lie derivatives. Then $\lambda$ is defined by means of a local natural operator $\hat{D}: \Gamma_{c}\left(\Lambda^{p_{1}} T^{*} M\right) \times \ldots \times \Gamma_{c}\left(\Lambda^{p_{k-1}} T^{*} M\right) \rightarrow \Gamma_{c}\left(\Lambda^{p_{k}} T M \otimes \Lambda^{m} T^{*} M\right)$. However, $\Lambda^{p_{k}} \mathbb{R}^{m} \otimes \Lambda^{m} \mathbb{R}^{m *} \simeq \Lambda^{m-p_{k}} \mathbb{R}^{m *}$ as $G L(m, \mathbb{R})$-spaces and so the corresponding natural vector bundles are naturally equivalent. Moreover, under this identification, the canonical pairing with values in the $m$-forms is just the wedge product.

What remains is to classify all local natural operators $D: \Gamma_{c}\left(\Lambda^{p_{1}} T^{*} M\right) \times \ldots \times$ $\Gamma_{c}\left(\Lambda^{p_{\ell}} T^{*} M\right) \rightarrow \Gamma_{c}\left(\Lambda^{q} T^{*} M\right), 0 \leq q \leq m$. This can be done very easily by the general technique described in 1.5. Let us sketch briefly how to do it. At the level of standard fibers of the jet spaces, we can write the operator in question as a linear combination of terms $\varphi_{i_{1} \ldots i_{p_{1}}, \alpha} \ldots \psi_{j_{1} \ldots j_{p_{\ell}, \beta}}$ denoting tensor product of derivatives of the tensors. The indices indicated by $\alpha, \ldots, \beta$ are symmetric, we have to alternate all indices at the end, and there are no indices to contract over, which implies that each individual term in the tensor product can be differentiated at most once. Thus, up to scalar multiples, the only way to get a local natural operator is to choose $n=q-\left(p_{1}+\cdots+p_{\ell}\right) \geq 0$ indices $1 \leq j_{1}<\ldots j_{n} \leq \ell$ and to define

$$
D_{j_{1}, \ldots, j_{n}}^{p_{1}, \ldots, p_{\ell}}\left(s_{1}, \ldots, s_{\ell}\right):=d s_{j_{1}} \wedge \cdots \wedge d s_{j_{n}} \wedge s_{i_{1}} \wedge \cdots \wedge s_{i_{\ell-n}}
$$

where $i_{1}, \ldots, i_{\ell-n}$ are the remaining indices among $\{1, \ldots, \ell\}$.

In words, the operators in question are built from the wedge products, exterior differentials and integration of $m$-forms. The classification in each concrete situation is a matter of simple combinatorics. Let us formulate two corollaries.

6.4. Corollary. Let $M$ be an arbitrary $m$-dimensional oriented smooth manifold, $m \geq 2$. All bilinear (not necessarily continuous) operators $D: \Gamma_{c}\left(\Lambda^{p_{1}} T^{*} M\right) \times$ $\Gamma_{c}\left(\Lambda^{p_{2}} T^{*} M\right) \rightarrow \Gamma\left(\Lambda^{q} T^{*} M\right), q>0, p_{1}<m, p_{2}<m$, commuting with the Lie derivatives are local natural operators (thus obtained by means of wedge product and exterior differential).

If we drop one of the assumptions that $p_{1}, p_{2}<m$ and $q>0$ then there are operators like

$$
\begin{gathered}
D: \Gamma_{c}\left(\Lambda^{m} T^{*} M\right) \times \Gamma_{c}\left(\Lambda^{p} T^{*} M\right) \rightarrow \Gamma\left(\Lambda^{p+1} T^{*} M\right), \quad(\omega, \varphi) \mapsto\left(\int_{M} \omega\right) d \varphi \\
D: \Gamma_{c}\left(\Lambda^{m-p} T^{*} M\right) \times \Gamma_{c}\left(\Lambda^{p} T^{*} M\right) \rightarrow C^{\infty}(M), \quad(\omega, \varphi) \mapsto \int_{M}(\omega \wedge \varphi) .
\end{gathered}
$$


6.5. Corollary. The space of all separately continuous $k$-linear operators

$$
C_{c}^{\infty}(M) \times \ldots \times C_{c}^{\infty}(M) \rightarrow C^{\infty}(M)
$$

is linearly generated by the elementary almost natural operators obtained by choosing pairwise disjoint $m$-tuples of indices $I_{j}=\left\{i_{1}^{j}<\cdots<i_{m}^{j}\right\}$ among $\{1, \ldots, k\}$, considering the $m$-forms $d f_{i_{1}^{j}} \wedge \cdots \wedge d f_{i_{m}^{j}}$, distributing some of the other functions to the $m$-forms in order to integrate them, and to multiply the result of the integrations with the remaining functions.

In particular, all symmetric separately continuous $k$-linear operators are the scalar multiples of the pointwise multiplication.

This corollary is closely related to one of the results in [de Wilde-Lecomte], where the last assertion of the corollary is proved without any continuity assumptions.

6.6. A more difficult task is to get similar complete descriptions for operations with arguments involving some contravariant indices. We shall restrict ourselves to some bilinear operations. First we reprove the completely algebraic characterization of the Lie bracket, cf. [de Wilde-Lecomte].

Theorem. Let $M$ be an oriented smooth manifold of dimension $\geq 2, D: \mathcal{X}_{c}(M) \times$ $\mathcal{X}_{c}(M) \rightarrow \mathcal{X}(M)$ a (not necessarily continuous) bilinear operator which maps compactly supported vector fields to vector fields and commutes with Lie derivatives. Then $D$ is a scalar multiple of the Lie bracket.

Proof. Since $T M$ has no absolutely invariant sections, $D$ must be an almost natural operator according to 6.2. Moreover $T^{*} M \otimes \Lambda^{m} T^{*} M$ has no absolutely invariant sections since the elements $\lambda \cdot \operatorname{Id}$ from the center of $G L(m, \mathbb{R})$ act by multiplication with a nonzero power of $\lambda$, and so $D$ must in fact be a local natural operator. Now assuming locality it is easy to prove uniqueness of the Lie bracket by means of the technique indicated in 1.5 (c.f. [Čap], [Krupka-Mikolášová] and [van Strien]).

6.7. The Schouten bracket. The Lie bracket is a special case of the so called Schouten bracket $D: \Gamma_{c}\left(S^{k} T M\right) \times \Gamma_{c}\left(S^{\ell} T M\right) \rightarrow \Gamma\left(S^{k+\ell-1} T M\right), k>0, l>0$, (or symmetric Schouten concomitant) which can be defined as the restriction of the canonical Poisson bracket on the symplectic manifold $T^{*} M$ to the fiberwise polynomial functions on $T^{*} M$ identified with sections of $S^{k} T M$ and $S^{\ell} T M$.

Since the natural bundle $S^{k} T M, k>0$, corresponds to a non-trivial irreducible representation of $G L(m, \mathbb{R})$, there are no absolutely invariant sections in the target of the operations in question. Thus 6.2 implies that the operator must be a linear combination of elementary almost natural operators. But since there are no absolutely invariant sections in $S^{k} T^{*} M \otimes \Lambda^{m} T^{*} M$, the operator must be local. Then the general procedure for the classification shows that there is a unique local operator, up to scalar multiples. Thus we have proved

Theorem. Let $M$ be an $m$-dimensional oriented smooth manifold, $m \geq 2$. Each bilinear operator $D: \Gamma_{c}\left(S^{k} T M\right) \times \Gamma_{c}\left(S^{\ell} T M\right) \rightarrow \Gamma\left(S^{k+\ell-1} T M\right), k>0, l>0$, which commutes with Lie derivatives is a scalar multiple of the Schouten bracket.

6.8. The Schouten-Nijenhuis bracket. Another generalization of the Lie bracket is the bracket $D: \Gamma_{c}\left(\Lambda^{p} T M\right) \times \Gamma_{c}\left(\Lambda^{q} T M\right) \rightarrow \Gamma_{c}\left(\Lambda^{p+q-1} T M\right)$ defined by 
the formula

$$
\begin{aligned}
& D\left(X_{1} \wedge \cdots \wedge X_{p}, Y_{1} \wedge \cdots \wedge Y_{q}\right)= \\
& \quad=\sum_{i, j}(-1)^{i+j}\left[X_{i}, Y_{j}\right] \wedge X_{1} \wedge \ldots^{\wedge_{i}} \ldots \wedge X_{p} \wedge Y_{1} \wedge \ldots{ }^{\wedge j} \ldots \wedge Y_{q} .
\end{aligned}
$$

This operation is called Schouten-Nijenhuis bracket and defines the structure of a graded Lie algebra on the space $\Lambda T M$.

Exactly as in 5.10, both the target bundle and $\Lambda^{q} T^{*} M \otimes \Lambda^{m} T^{*} M$ admit no absolute invariant sections and so all operators of the given type which commute with Lie derivatives (without any continuity assumption) must be local. But this implies, cf. [Michor 1],

Theorem. Let $M$ be an $m$-dimensional oriented smooth manifold, $m \geq 2$. Each bilinear operator $D: \Gamma_{c}\left(\Lambda^{p} T M\right) \times \Gamma_{c}\left(\Lambda^{q} T M\right) \rightarrow \Gamma\left({ }^{p+q-1} T M\right), p>0, q>0$, which commutes with Lie derivatives is a scalar multiple of the Schouten-Nijenhuis bracket.

6.9. The Frölicher-Nijenhuis bracket. Consider the first order natural vector bundles $\Lambda^{k} T^{*} \otimes T$. Sections of values of such a natural bundle are usually called vector valued $k$-forms, and the space of these sections over $M$ is denoted by $\Omega^{k}(M, T M)$. We shall give a completely algebraic characterization of a bracket which is closely related to the Frölicher-Nijenhuis bracket.

For any $M$ the bundle $\Lambda T^{*} M \otimes T M=\oplus_{k} \Lambda^{k} T^{*} M \otimes T M$ is a bundle of graded modules over the bundle of graded commutative algebras $\Lambda T^{*} M$, where the action is induced by the wedge product. Moreover there is an absolutely invariant section $\mathbb{I}$ of $T^{*} M \otimes T M$, which corresponds to the identity map on $T M$. By acting on this element we can view $\Lambda^{k-1} T^{*} M$ as a subbundle of $\Lambda^{k} T^{*} M \otimes T M$. This subbundle is actually a direct summand, and a projection $\Lambda^{k} T^{*} M \otimes T M \rightarrow \Lambda^{k-1} T^{*} M$ is induced by mapping $\omega \otimes X$ to $\frac{(-1)^{k-1}}{m-k+1} i_{X} \omega$, where $i_{X}$ denotes the usual insertion operator. The kernel of this projection is again a natural vector bundle $C^{k}$, whose sections are called trace free vector valued $k$-forms. We shall write briefly $\mathcal{C}^{k}(M)$ for the space of these sections. (The decomposition of $\Lambda^{k} T^{*} M \otimes T M$ constructed above corresponds exactly to the decomposition of the representation $\Lambda^{k} \mathbb{R}^{m *} \otimes \mathbb{R}^{m}$ of $G L(m, \mathbb{R})$ into irreducible representations.)

There is a bilinear natural operator [ , ] : $\Lambda^{k} T^{*} \otimes T \times \Lambda^{\ell} T^{*} \otimes T \rightarrow \Lambda^{k+\ell} T^{*} \otimes T$ called the Frölicher-Nijenhuis bracket, which defines a graded Lie algebra structure on the space of vector valued differential forms and plays an important role in the theory of generalized connections (c.f. [Frölicher-Nijenhuis] and [Michor 2]). Using the projections onto trace free vector valued forms constructed above we can compress this bracket to a bilinear natural operator [ , $]^{c}: \mathcal{C}^{k}(M) \times \mathcal{C}^{\ell}(M) \rightarrow$ $C^{k+\ell}(M)$ for any smooth manifold $M$. It turns out that this is again a graded Lie bracket (c.f. [Michor-Schicketanz]).

6.10. Theorem. Let $M$ be an oriented smooth manifold of dimension $m \geq 2$, $D: \mathcal{C}_{c}^{k}(M) \times \mathcal{C}_{c}^{\ell}(M) \rightarrow \mathcal{C}^{k+\ell}(M)$ a not necessarily continuous bilinear operator which commutes with Lie derivatives. Then $D$ is a scalar multiple of the compression of the Frölicher-Nijenhuis bracket.

Proof. First note that $\mathcal{C}^{k+\ell}$ has no nonzero absolutely invariant sections since it corresponds to an irreducible representation of $G L(m, \mathbb{R})$. Thus by $6.2 D$ must be 
an almost natural operator. Moreover for $k<m$ there are no absolutely invariant sections of $C^{k *} M \otimes \Lambda^{m} T^{*} M$, since scalar multiples of the identity act nontrivially. But $\mathcal{C}^{m}(M)=0$ since $\Lambda^{m} \mathbb{R}^{m *} \otimes \mathbb{R}^{m} \simeq \Lambda^{m-1} \mathbb{R}^{m *}$ (c.f. [Michor-Schicketanz]). Thus $D$ must be a local operator. Now using the projections on the left hand side and the inclusion into all vector valued differential forms on the right hand side, $D$ induces a local natural operator $\tilde{D}: \Omega^{k}(M, T M) \times \Omega^{\ell}(M, T M) \rightarrow \Omega^{k+\ell}(M, T M)$. But these operators have been completely classified in [Koláŕ-Michor] and [Čap]. Looking at the list of possible operators and taking into account that the values must be trace free and the operator depends only on the trace free parts of the arguments one sees that in fact it must be a scalar multiple of the Frölicher-Nijenhuis bracket, so the result follows.

\section{REFERENCES}

Cahen, M.; de Wilde, M.; Gutt, S., Local cohomology of the algebra of $C^{\infty}$-functions on a connected manifold, Lett. Math. Phys. 4 (1980), 157-167.

C̆ap, A., All linear and bilinear natural concomitants of vector valued differential forms, Comment. Math. Univ. Carolinae 31,3 (1990), 567-587.

Čap, A.; Slovák, J., Infinitesimally natural operators are natural, Diff. Geom. and its Appl. vol 2 (1990), 45-55.

de Wilde, M.; Lecomte, P., Algebraic characterization of the algebra of functions and of the Lie algebra of vector fields on a manifold, Compositio Math. 45 (1982), 199-205.

Eck, D.J., Natural Sheafs, Illinois J. Math. 31, no. 2 (1987), 200-207.

Epstein, D.B.A.; Thurston, W.P., Transformation groups and natural bundles, Proc. London Math. Soc. 38 (1979), 219-236.

Frölicher, A.; Kriegl, A., Linear spaces and differentiation theory, Pure and Applied Mathematics, J. Wiley, Chichester, 1988.

Frölicher, A.; Nijenhuis, A., Theory of vector valued differential forms, Part I, Indagationes Math. 18 (1956), 338-359.

Hörmander,L., The Analysis of Linear Partial Differential Operators I, Grundlehren 256, Springer, Berlin New York, 1983.

Kirillov, A.A., Invariant operators over geometrical quantities, (Russian), Current problems of mathematics, Vol 16, VINITI, 1980, pp. 3-29.

Kolář, I.; Michor, P.W., All natural concomitants of vector valued differential forms, Proc. Winter School on Geometry and Physics, Srni 1987, Supp. ai Rend. Circolo Math. di Palermo II-16 (1987), 101-108.

Kolář, I.; Michor, P.W.; Slovák, J., Natural Operations in Differential Geometry, Springer, Berlin New York, 1993.

Krupka, D.; Mikolášová, V., On the uniqueness of some differential invariants: d, [ , ], $\nabla$, Czechoslovak Math. J. 34 (1984), 588-597.

Michor, P.W., Remarks on the Schouten-Nijenhuis bracket, Supp. ai Rend. Circolo Math. di Palermo, Serie II 16 (1987), 207-215.

Michor, P.W., Remarks on the Frölicher-Nijenhuis bracket, Proc. of the Conf. on Diff. Geom. and its Appl., Brno 1986, D. Reidl, 1987.

Michor, P.W.; Schicketanz, H., A cohomology for vector valued differential forms, Ann. Global Anal. Geom. 7,3 (1989), 163-169.

Palais, R. S.; Terng, C. L., Natural bundles have finite order, Topology 16 (1977), 271-277.

Samelson, H., Notes on Lie algebras, Universitext, Springer-Verlag, 1989.

Slovák, J., Peetre theorem for nonlinear operators, Annals of Global Analysis and Geometry 6 (1988), 273-283.

Terng C. L., Natural vector bundles and natural differential operators, American J. of Math. 100 (1978), 775-828.

van Strien, S., Unicity of the Lie product, Compositio Math. 40 (1980), 79-85.

Institut für Mathematik, Universität Wien, Strudlhofgasse 4, 1090 Wien, Austria 
and Erwin Schrödinger International Institute for Mathematical Physics, Pasteurgasse 6/7, 1090 Wien, Austria

Department of Algebra and Geometry, Masaryk University, JanáčKovo nám. 2A 66295 Brno, Czech Republic 